\title{
Hacia una caracterización empírica del lenguaje esquizofrénico: de la descripción estructural de los discursos a la reconstrucción de los procesos implicados en la producción verbal desviada
}

\section{Mercedes Belinchón}

\author{
Universidad Autónoma de Madrid
}

\section{INTRODUCCION}

Hace más de ochenta años que los psicólogos y psiquiatras, mediante el uso de conceptos tales como los de «esquizofrenia" y "psicosis», han tratado de acotar y definir de un modo científicamente útil (Zubin y Spring, 1977) uno de los fenómenos más antiguos, universales y sorprendentes de la especie humana: la locura. Desde entonces, movidos quizá por la misma mezcla de asombro, miedo y fascinación que lleva a los miembros de ciertas culturas a hacer del demente un miembro marginal de la comunidad $y / o$ a investirle con atributos mágicos (Bastide, 1976), los profesionales de la salud mental y los investigadores de la conducta humana han tratado de encontrar explicaciones satisfactorias a la conducta peculiar de los psicóticos y han buscado, desde diferentes perspectivas y marcos teóricos, sistemas y procedimientos capaces de describir, predecir, controlar y/o extirpar la enfermedad.

En su interpretación más ancestral (y también en su interpretación más popular y común) la locura ha sido, indudablemente, reificada, dotada de entidad: se la ha considerado como equivalente a «algo» (desconocido y quizá mágico), que «ocurre» en el interior de los sujetos, que escapa al control de quien lo sufre, y que, por lo general, puede ser reconocido por los no enfermos en ciertos tipos de conductas y/o verbalizaciones.

Según observan Harrow y Quinlan (1985), la «reificación» que subyacía a las definiciones no científicas de la locura fue tácitamente asumida, desde sus inicios, tanto por la Psicología como por la Psiquiatría moderna. La identificación de la enfermedad con un elemento mórbido al que se denominó «desorden formal de pensamiento», en las primeras definiciones «técnicas» de la esquizofrenia (Bleuler, 1911; Kraepelin, 1919), y la vinculación inferencial que a partir de esta identificación se estableció en el contexto psiquiátrico entre la observación de alteraciones severas en el uso comunicativo del lenguaje de los pacientes, la existencia de un desorden del pensamiento y el diagnóstico de esquizofrenia, pronto hicieron de la descripción de la conducta lingǘstica una de las bases más sólidas del diagnóstico clínico ( «To be a good clinician one must be a good listener» rezaba, por ejemplo, un viejo proverbio recogido por Harrow y Quinlan, 1985, pág. 209). Paralelamente, y a medida que fue desarrollándose la propia Psicología científica, el análisis teórico y empírico de las alteraciones del lenguaje fue dando paso a la elaboración de múltiples teorías e hipótesis sobre la esquizofrenia, sobre sus trastornos 
de pensamiento más patognomónicos y, evidentemente también, sobre las propias alteraciones verbales que fundamentaban su diagnóstico clínico.

El supuesto bleuleriano de especificidad diagnóstica del «desorden formal de pensamiento» (que, indirectamente, llevaba a considerar ciertas alteraciones del discurso verbal - tales como la «incoherencia», los «descarrilamientos de ideas», los «neologismos», etc. - como rasgos propios de un «lenguaje esquizofrénico») dio paso a una cantidad ingente de investigaciones que, utilizando definiciones clínicas y/o índices psicolingüísticos trataron de verificar la existencia de alteraciones del lenguaje específicamente esquizofrénicas y, en última instancia, la existencia de déficit cognitivos y/o lingüísticos capaces de fundamentar conductualmente los constructos teóricos elaborados por Bleuler en Demencia Precoz (1911).

Los estudios realizados en el contexto psiquiátrico han demostrado empíricamente, con relativa consistencia, que algunos de los rasgos que tradicionalmente se han considerado como patognomónicos del lenguaje de los esquizofrénicos (vg. la incoherencia, los neologismos, las sustituciones bizarras de palabras) constituyen una proporción relativamente pequeña de la producción verbal de estos sujetos y aparecen también (e incluso con una frecuencia similar) en otros tipos de psicosis - fundamentalmente, la manía- (cfr. Andreasen, 1979a, 1979b; Andreasen y Grove, 1979; Andreasen et al., 1985; Harrow y Quinlan, 1975; Siegel et al., 1976; Grove y Andreasen, 1985; Harrow y Quinlan, 1985). Algunos estudios de seguimiento (vg. Flekkoy, 1975; Siegel et al., 1976; Grove y Andreasen, 1985; Harrow y Quinlan, 1985) han confirmado, además, observaciones no sistemáticas de las primeras descripciones clínicas relativas al carácter episódico, cíclico e intermitente de las alteraciones esquizofrénicas del lenguaje y a su extraordinaria variabilidad inter e intraindividual (Séglas, 1982; Bleuler, 1911; Kraepelin, 1919; M. Bleuler, 1982).

En el ámbito de las investigaciones experimentales, por otro lado, los resultados obtenidos hasta el momento son también heterogéneos y no siempre convergentes en lo que respecta a la posibilidad de identificar, en el lenguaje de los esquizof rénicos, un conjunto definido, estable y específico de alteraciones.

Trabajos de factura teórica tan distinta como los de Chapman, Chapman y Miller (1964), Cohen y cols. (1967; 1974, 1978), Salzinger y cols. (1964, 1978, 1980), Tucker y Rosenberg $(1975,1979)$, Rochester y cols. $(1977,1979)$, Manschreck y cols. (1979, 1980, 1981, 1984), Hoffman y cols. (1982, 1984, y en prensa), Chaika y Alexander (1982) y Fraser et al. (1986) - por citar los más representativos - han aportado evidencia congruente con la hipótesis de que, cuando son comparados con sujetos normales, los esquizofrénicos muestran perturbaciones severas relacionadas con la planificación, codificación y realización lingüística de sus discursos (especialmente en lo que respecta a los componentes semántico y pragmático del procesamiento). Sin embargo, sólo en muy pocos estudios (vg. Hoffman et al., 1982, y en prensa), los análisis cuantitativos y/o cualitativos de las producciones lingüísticas (espontáneas o elicitadas) han permitido diferenciar a los esquizofrénicos de orros grupos psicóticos tales como los maníacos o los pacientes (no esquizofrénicos) con «desorden formal de pensamiento» y han proporcionado un cuadro descriptivo consistente del llamado «lenguaje esquizofrénico» (cfr. revisiones anteriores nuestras en este mismo volumen y en Ruiz Vargas, 1987).

Pese a su heterogeneidad teórica y metodológica, la mayoría de estas investigaciones ha permitido, no obstante, la formulación de hipótesis explicativas plausibles que vinculan los déficit lingüísticos de los esquizofrénicos con alteraciones funcionales de los sistemas responsables del procesamiento lingüístico y/o con déficit más generales en el sistema de procesamiento de la información (cfr. revisiones ya citadas). 
La confirmación de que ciertos índices psicolingüísticos pueden ser útiles para el diagnóstico diferencial de estos pacientes y para la elaboración de hipótesis comprensivas y científicas de la esquizofrenia ha sido estadísticamente verificada, ciertamente, en algunos estudios experimentales. Sin embargo, existen algunos puntos oscuros en estas investigaciones que impiden aceptar todavía, sin reservas importantes, tanto la hipótesis de que los esquizofrénicos sufren un tipo específico de perturbación lingüística como la hipótesis de que tal perturbación o déficit no tiene un carácter meramente episódico, relacionado con las fases agudas del trastorno.

La heterogeneidad metodológica de las investigaciones anteriores en lo que respecta a la selección de los grupos experimentales y de las muestras lingüísticas utilizadas en las comparaciones de los grupos (ver tabla I), la fragilidad de algunos de los índices lingüísticos utilizados (vg. su extrema dependencia respecto a variables clínicas como el subtipo diagnóstico o la cronicidad de la enfermedad en los pacientes), la comparación habitual -aunque inadecuada- de resultados obtenidos en tareas de tipo monológico y dialógico (que comportan exigencias comunicativas y cognitivas claramente diferenciadas -cfr. Mayor, 1984-), y la utilización, finalmente, de índices sintácticos, semánticos y pragmáticos en estudios que, en ningún caso, han sido diseñados como metodológicamente cruciales (es decir, capaces de discernir entre varias hipótesis alternativas), plantean problemas importantes que lievan a pensar que, desde un punto de vista metodológico, la evidencia empírica sobre la que algunos autores sostienen la hipótesis de que, en la esquizofrenia, existen déficit específicos que afectan a la producción verbal de los discursos, resulte débil en su conjunto.

La heterogeneidad misma de los marcos teóricos utilizados como referencia para el diseño de los trabajos empíricos de comparación lleva a considerar asimismo como imprescindible la realización de estudios metodológicos que posibiliten la «integración teórica» de los hallazgos empíricos obtenidos hasta la fecha y que permitan, en última instancia, la formulación de hipótesis explicativas compatibles con los modelos teóricos de producción verbal que en la actualidad son objeto de contrastación empírica en el contexto de la Psicología del Lenguaje (cfr. vg. Foss y Hakes, 1978; Butterworth, 1980; Van Dijk, 1980; Zammuner, 1981; Santacruz, 1984; García-Albea, Igoa y Sánchez Bernardos, 1987; Belinchón, en prensa).

El trabajo que presentamos a continuación constituye un primer acercamiento empírico a este tipo de cuestiones y surge, en primer lugar, como respuesta al hecho de que a pesar de su uso habitual como índice diagnóstico en el contexto clínico, y a pesar también de los numerosos estudios experimentales realizados hasta la fecha, los investigadores carecen tanto de una definición objetiva del llamado «lenguaje esquizofrénico» como de una teoría común que explique y/o prediga sus manifestaciones más frecuentes.

Realizado en un contexto teórico próximo a la Psicolingüística de orientación textual, el presente estudio se dirigirá, en primer lugar, a la comprobación de la hipóresis de que el lenguaje de los sujetos esquizofrénicos, analizado en términos estructurales, puede presentar rasgos o peculiaridades con significación diagnóstica (esto es, capaces de diferenciar a los esquizofrénicos de sus controles). En segundo lugar, y adoptando una perspectiva más metodológica, el trabajo asumirá la elaboración de índices lingüísticos de análisis que, aparte de su valor para la diferenciación empírica de los discursos desviados y normales, permitan una «reconstrucción» (siquiera aproximativa) de los procesos implicados en la producción de ambos tipos de discursos. Finalmente, y en conexión con los dos objetivos anteriores, se discutirán las vinculaciones funcionales que puedan detectarse entre las variables lingüisticas 
Ejemplos de criterios de selección de sujetos, tareas y muestras lingüísticas, en otros estudios sobre el discurso de los esquizofrénicos

\begin{tabular}{|c|c|c|c|}
\hline Estudio & Sujetos & Tarea & $\begin{array}{c}\text { Criterio de } \\
\text { selección de los textos } \\
\end{array}$ \\
\hline $\begin{array}{l}\text { Rochester y Martia } \\
\text { (1979). }\end{array}$ & $\begin{array}{l}10 \text { esquizof rénicos } \\
\text { con DFP. } \\
10 \text { esquizof rénicos } \\
\text { sin DFP. } \\
10 \text { normales. }\end{array}$ & $\begin{array}{l}\text { - Entrevista no es- } \\
\text { tructurada. } \\
\text { - Narración de un } \\
\text { texto previamen- } \\
\text { te leído. } \\
\text { - Descripción y ex- } \\
\text { plicación de his- } \\
\text { torietas. }\end{array}$ & $\begin{array}{l}\text { - Muestras de } 3 \text { min. } \\
\text { de duración ininte- } \\
\text { rrumpida. } \\
\text { - } 15 \text { oraciones inde- } \\
\text { pendientes. }\end{array}$ \\
\hline $\begin{array}{l}\text { Hoffman et al. } \\
(1982) .\end{array}$ & $\begin{array}{l}11 \text { esquizof rénicos. } \\
9 \text { psicóticos no es- } \\
\text { quizofrénicos. }\end{array}$ & $\begin{array}{l}\text { - Entrevista no } \\
\text { psiquiátrica. }\end{array}$ & $\begin{array}{l}\text { - Respuestas con más } \\
\text { de cinco oraciones, } \\
\text { sin alteración de } \\
\text { contenido. }\end{array}$ \\
\hline Harvey (1983). & $\begin{array}{l}12 \text { esquizo frénicos } \\
\text { agudos con DFP. } \\
10 \text { esquizof rénicos } \\
\text { agudos sin DFP. } \\
10 \text { maniacos con } \\
\text { DFP. } \\
10 \text { maniacos sin } \\
\text { DFP. } \\
10 \text { normales. }\end{array}$ & $\begin{array}{l}\text { - Entrevista psi- } \\
\text { quiátrica. } \\
\text { - Conversación so- } \\
\text { bre tema libre. }\end{array}$ & $\begin{array}{l}\text { - Respuestas comple- } \\
\text { tas. }\end{array}$ \\
\hline $\begin{array}{l}\text { Allen y Allen (1983, } \\
1984,1985) \text {. }\end{array}$ & $\begin{array}{l}10 \text { esquizof rénicos } \\
\text { positivos. } \\
10 \begin{array}{l}\text { esquizof rénicos } \\
\text { negativos. } \\
9 \text { normales }\end{array}\end{array}$ & $\begin{array}{l}\text { - Descripciones lá- } \\
\text { minas T. A. }\end{array}$ & $\begin{array}{l}\text { - } 2 \text { minutos de res- } \\
\text { puesta por lámina } \\
\text { (total } 8 \text { minutos). }\end{array}$ \\
\hline $\begin{array}{l}\text { Reich y Cutting } \\
(1982) \text {. }\end{array}$ & $\begin{array}{l}25 \text { esquizofrénicos. } \\
20 \text { depresivos. } \\
32 \text { lesiones cerebra- } \\
\text { les. } \\
20 \text { normales. }\end{array}$ & $\begin{array}{l}\text { - Descripción de } \\
\text { una lámina. }\end{array}$ & $\begin{array}{l}-2 \text { minutos de res- } \\
\text { puesta. }\end{array}$ \\
\hline $\begin{array}{l}\text { Chaika, Lamba y } \\
\text { Alexander (1982). }\end{array}$ & $\begin{array}{l}22 \text { esquizof rénicos y } \\
\text { psicóticos con al- } \\
\text { teraciones del } \\
\text { lenguaje. }\end{array}$ & $\begin{array}{l}\text { - Narración de una } \\
\text { película. }\end{array}$ & $\begin{array}{l}\text { - Respuestas comple- } \\
\text { tas. }\end{array}$ \\
\hline Rutter (1985). & $\begin{array}{l}35 \text { esquizof rénicos. } \\
7 \text { pacientes con } \\
\text { trastornos efecti- } \\
\text { vos. } \\
10 \text { normales. }\end{array}$ & $\begin{array}{l}\text { - Explicación mo- } \\
\text { tivo de ingreso. } \\
\text { - Conversación di- } \\
\text { rigida. }\end{array}$ & $\begin{array}{l}\text { - Primeras } 10 \text { oracio- } \\
\text { nes de la respuesta. } \\
\text { - Primeras } 20 \text { emisio- } \\
\text { nes. }\end{array}$ \\
\hline Praser et al. (1986) & $\begin{array}{l}50 \text { esquizof rénicos } \\
\text { agudos. } \\
51 \text { maniacos. }\end{array}$ & $\begin{array}{l}\text { - Explicación mo- } \\
\text { tivo de ingreso. } \\
\text { - Descripción de } \\
\text { una lámina. }\end{array}$ & $\begin{array}{l}\text { - Primeras } 1.000 \text { pala- } \\
\text { bras. }\end{array}$ \\
\hline
\end{tabular}


utilizadas en el análisis de los discursos y otras variables (fundamentalmente cognitivas) que, en otros estudios, han dado pie a la formulación de hipótesis explicativas del déficit lingüístico esquizofrénico.

Asumiendo, pues, la idea de que para lograr una buena adecuación explicativa de la actividad lingüística (en nuestro caso, perturbada) es necesario disponer, en primer lugar, de una suficiente adecuación descriptiva (Garcia-Albea, 1986), y asumiendo también el supuesto de que las descripciones micro y macroestructurales de los textos o discursos pueden proporcionar indicadores de interés respecto a los mecanismos y los sesgos de la actividad que los genera (De Beaugrande, 1979; Levy, 1980), se realizó un análisis lingüístico multinivel de textos producidos por sujetos esquizofrénicos y normales al objeto de obtener evidencia relevante para la clarificación empírica de tres tipos específicos de hipótesis:

En primer lugar, de la hipótesis que asume que, a partir del análisis de los discursos verbales, es posible identificar marcadores o índices lingüísticos capaces de diferenciar entre grupos de sujetos esquizofrénicos y normales, aun en el caso de que el primero sea un grupo clínicamente heterogéneo.

En segundo lugar, de la hipótesis de que, a partir del análisis teórico de los índices lingüísticos con capacidad discriminativa más alta, es posible inferir cuáles son los componentes y/o los niveles de procesamiento lingüístico que están más afectados en los sujetos esquizofrénicos.

En tercer y último lugar, de la hipótesis de que, empíricamente, es posible establecer relaciones de covariación entre las alteraciones esquizofrénicas de lenguaje y las alteraciones que supuestamente estos pacientes sufren en otros procesos cognitivos tales como la atención o la habilidad para solucionar problemas de ordenación lógica. En la medida en que, teóricamente, estas dos habilidades están relacionadas funcionalmente con la actividad lingüística de producción textual (De Beaugrande, 1980), la identificación empírica de tales relaciones podría interpretarse como una base razonable y sólida para la generación de hipótesis explicativas del déficit lingüistico esquizof rénico y/o, en su caso, para la formulación de una caracterización objetiva y científica del déficit cognitivo esquizofrénico en su conjunto.

La observación, recogida en trabajos anteriores (vg. Pavy, 1968; Zavarin, 1977, 1980; Chaika, 1982; Belinchón, 1987), de que, considerada en términos psicolingüísticos, la alteración de los esquizofrénicos parece afectar más claramente a la producción y organización de unidades lingüísticas complejas (como el discurso o la conversación) que a la producción de unidades simples, constituye la razón principal que nos ha impulsado a seleccionar, como nivel prioritario del análisis lingüístico, el nivel de discurso y no el nivel oracional o morfoléxico. La sospecha (planteada explícitamente por Mancuso et al., 1982) de que la esquizofrenia puede no ser una variable intermedia útil en la investigación psicolingüística de los discursos desviados, constituye, por otro lado, la razón fundamental que explica la heterogeneidad clínica del grupo de esquizofrénicos utilizado en este estudio.

\section{METODO}

\section{Sujetos}

La investigación fue realizada con un total de 40 sujetos: 20 pacientes diagnosticados de esquizofrenia y psicosis esquizofreniforme (según la DSM-III), ingresados en la Unidad de Psiquiatría del Hospital Civil de Bilbao (Basurto), y 20 sujetos normales (es decir, sin antecedentes psiquiátricos). 


\section{TABLA II}

Caracteristicas de los sujetos esquizofrénicos

\begin{tabular}{lcclcccl}
\hline Nombre & Edad & Sexo & Estudios & $\begin{array}{c}\text { Ingresos } \\
\text { previos }\end{array}$ & $\begin{array}{c}\text { Primer } \\
\text { ingreso }\end{array}$ & $\begin{array}{c}\text { Días } \\
\text { hospital }\end{array}$ & Dosis media diaria de medición \\
\hline 1) Eduardo A. & 21 & V & F. P. & 1 & 1984 & 5 & $24 \mathrm{mg}$ (Haloperidol). \\
2) José Manuel P. & 30 & V & E. G. B. & 0 & - & 4 & $20 \mathrm{mg}$ (Haloperidol). \\
3) Almudena B. & 22 & M & Turismo & 0 & - & 10 & Ninguna \\
4) Teresa M. & 23 & M & Esteticista & 0 & - & 20 & $?$ \\
5) José María A. & 25 & V & B. U. P. & 1 & - & 2 & $10 \mathrm{mg}$ (Haloperidol)+60 mg 2-cl-11-Dibenzanotiazepina (Etu- \\
6) José María S. & 22 & V & C. E. P. & 0 & - & 7 & mina). \\
7) Juan Manuel E. & 21 & V & F. P. & 0 & - & 19 & $24 \mathrm{mg}$ (Haloperidol)+16 mg (Pimozida-Orap). \\
8) María Victoria B. & 22 & M & F. P. & 0 & - & 15 & $23 \mathrm{mg}$ (Haloperidol). \\
9) Juan Carlos D. & 24 & V & F. P. & 1 & 1984 & 10 & $16 \mathrm{mg}$ (Haloperidol). \\
10) Javier J. & 17 & V & C. E. P. & 2 & 1985 & 10 & $7 \mathrm{mg}$ (Haloperidol)+25 mg (Clorpromazina-Largactil). \\
11) Aitor S. & 20 & V & F. P. & 0 & - & 5 & $10 \mathrm{mg}$ (Haloperidol)+200 mg (Clorpromazina-Largactil). \\
12) José Luis P. & 22 & V & E. G. B. & 1 & 1982 & 12 & $17 \mathrm{mg}$ (Haloperidol)+40 mg 2-cl-11-Dibenzanotiazepina (Etu- \\
13) José Antonio P. & 22 & V & E. G. B. & 0 & - & 4 & $\mathrm{mina).}$ \\
14) Isidro T. & 20 & V & E. G. B. & 1 & 1986 & 5 & $14 \mathrm{mg}$ (Haloperidol). \\
15) Francisco R. & 27 & V & E. G. B. & 1 & 1983 & 9 & $19 \mathrm{mg}$ (Haloperidol). \\
16) Mari Carmen H & 20 & M & Aux. enf. & 0 & - & 20 & $4 \mathrm{mg}$ (Haloperidol). \\
17) Nieves S. E. & 27 & M & C. E. P. & 0 & - & 3 & $12 \mathrm{mg}$ (Haloperidol)+60 mg 2-cl-11-Dibenzanotiazepina (Etu- \\
18) José Antonio L. & 22 & V & F. P. & 1 & 1985 & 5 & $\mathrm{mina).}$ \\
19) Orlando V. & 18 & V & E. G. B. & 0 & - & 11 & $?$ \\
20) Mari Carmen E. & 24 & M & F. P. & 1 & 1985 & 9 & $16 \mathrm{mg}$ (Pimozida-Orap forte). \\
& & & & & & & 20 mg (Haloperidol). \\
& & & & & & $45 \mathrm{mg}$ (Cloperacepato dipotásico-Tranxilium).
\end{tabular}


Los pacientes (14 hombres y 6 mujeres) fueron seleccionados a partir de una primera muestra de 33 sujetos psicóticos de ambos sexos. Las variables que determinaron su inclusión definitiva en el grupo experimental fueron: a) su diagnóstico clínico; b) su edad; c) la duración previa de su enfermedad, y d) su puntuación en la Escala Verbal de WAIS.

Como se refleja en las tablas II y III, los sujetos seleccionados constituían un grupo de edad relativamente joven $(\bar{x}=22,45$ años; rango $=17-30$ años) y clínicamente heterogéneo. En 11 de los casos ( 55 por 100 del total), el actual era su primero ingreso en una institución psiquiátrica, en 8 de los casos (40 por 100) era el segundo, y en tan sólo un caso (5 por 100) era el tercero. Los pacientes que habían recibido terapia electroconvulsiva y los que, además de un cuadro conductual de esquizofrenia, presentaban un cuadro de toxicomanía y/o lesión cerebral fueron excluidos de la muestra. Los sujetos con puntuaciones inferiores a 90 en la escala verbal del WAIS fueron también excluidos. Todos los pacientes habían nacido en el País Vasco o residian allí desde su primera infancia. Aunque algunos de ellos entendían o hablaban el euskera, ninguno era bilingïe.

La participación de los sujetos en el estudio fue totalmente voluntaria y tuvo lugar, en todos los casos, entre el segundo y el vigésimo día de su estancia en la unidad de agudos del hospital ( $\bar{x}=9,3 ; s d=5,6$ días). Los datos, sin embargo, sólo fueron analizados cuando se completó y confirmó el diagnóstico clínico. En el momento de realizar las pruebas, todos los sujetos menos uno recibían medicación antipsicótica. Como han demostrado Hymowitz y Spohn (1980), aunque este tipo de medicación reduce el estado de agitación general de los sujetos, no parece disminuir ni la complejidad estructural ni el grado de coherencia del habla de los pacientes.

TABLA III

Clasificación clínica de los esquizofrénicos de la muestra

\begin{tabular}{|c|c|c|c|c|}
\hline \multirow[b]{2}{*}{ Pacient. N.o } & \multicolumn{4}{|c|}{ Valoración en dimensiones clínicas } \\
\hline & Parano. & Sintomatología & Ajuste premórbido & DFP \\
\hline 1 & Paranoide & Positiva & Bueno & No \\
\hline 2 & Mixto & Mixta & Bueno & Sí \\
\hline 3 & Mixto & Negativa & Bueno & No \\
\hline 4 & Mixto & Mixta & Bueno & No \\
\hline 5 & Mixto & Negativa & Malo & No \\
\hline 6 & Mixto & Negativa & Malo & No \\
\hline 7 & No paranoide & Mixta & Bueno & Sí \\
\hline 8 & Paranoide & Positiva & Bueno & No \\
\hline 9 & Mixto & Mixta & Malo & Sí \\
\hline 10 & Mixto & Mixta & Malo & Sí \\
\hline 11 & Mixto & Negativa & Malo & Sí \\
\hline 12 & Paranoide & Positiva & Bueno & No \\
\hline 13 & Mixto & Positiva & Bueno & Sí \\
\hline 14 & Mixto & Mixta & Bueno & No \\
\hline 15 & Mixto & Mixta & Malo & Sí \\
\hline 16 & Mixto & Negativa & Bueno & Sí \\
\hline 17 & Paranoide & Mixta & Bueno & No \\
\hline 18 & Mixto & Positiva & Bueno & No \\
\hline 19 & Mixto & Mixta & Malo & No \\
\hline 20 & Mixto & Negativa & Bueno & Sí \\
\hline
\end{tabular}

El grupo de control estuvo constituido por 20 sujetos normales voluntarios ( 6 de ellos, celadores y auxiliares de clínica del propio hospital), igualados uno a uno con los pacientes en edad, sexo, nivel sociocultural y comuni- 
dad lingüística de origen. A pesar de las precauciones tomadas en la selección de este grupo (vg. eliminar de la muestra aquellos controles cuya puntuación en el WAIS fuera superior a la más alta alcanzada en el grupo de esquizofrénicos), no fue posible evitar una diferencia significativa de alrededor de 10 puntos entre los grupos en esta variable, favorable a los normales $(p<.01)$. Esta diferencia, absolutamente congruente con los resultados de las investigaciones psicométricas de la inteligencia de los esquizof rénicos (cfr. Aylward, Walkes y Bettes, 1984), fue objeto de control estadístico en el análisis de los resultados que se presentará más adelante.

\section{Materiales y pruebas}

Todos los sujetos (esquizofrénicos y controles) de la presente investigación fueron objetos de una evaluación compleja de carácter clínico, psicométrico y experimental, orientada a obtener información tanto de sus características diagnósticas como de su ejecución en las variables lingüísticas y cognitivas objeto del estudio.

Para la evaluación clínica de los sujetos, se utilizaron los criterios DSMIII y dos escalas de aplicación individual: la B.P.R.S. (Overall y Gorham, 1962), para evaluar el nivel de severidad global de la sintomatología y la T.L.C. (Andreasen, 1979a, 1979b) para la evaluación del «desorden formal del pensamiento». La adaptación al castellano de esta última escala corrió a cargo de la profesora Carmen Vizcarro (U.A.M.). La traducción de la B.P.R.S. fue realizada por el equipo médico del Servicio de Psiquiatría del Hospital de Basurto (profesores doctores José Guimón y Jorge Grijalbo). Su administración, así como el diagnóstico clínico de los sujetos, fue realizado íntegramente por el doctor Grijalbo.

La evaluación del nivel de inteligencia verbal de los sujetos (que sirvió para la selección definitiva de los grupos) se realizó a partir de la administración de la Escala Verbal del WAIS (adaptación española, T.E.A., 1970). Complementariamente, y al objeto de poder evaluar también algunos aspectos específicos del funcionamiento intelectual de los pacientes (como la capacidad para ordenar lógicamente una serie de estímulos significativos - solución de problemas -), se administró la subescala de «Historietas» de la Escala Manipulativa de la misma prueba.

La valoración del déficit atencional de los sujetos (única variable cognitiva experimental utilizada en nuestro estudio) se realizó a través de una versión «A-X» del «Test de Ejecución Continua» («Continuous Performance Test» -C.P.T.—, diseñado originalmente por Rosvold et al., 1956). Dicha versión fue elaborada ex profeso para este estudio por el profesor José María Ruiz Vargas (Facultad de Psicología, U.A.M.). La presentación de los estímulos y la tabulación de las respuestas se realizó mediante un microordenador Sinclair, modelo ZX Spectrum-48 K, con teclado de burbuja. Como pantalla, se utilizó un monitor de TV en blanco y negro de 16 pulgadas. La codificación del programa de la tarea en lenguaje Basic fue realizada por el profesor José Luis Zaccagnini (Facultad de Psicología, U.A.M.).

Las muestras lingüisticas del estudio fueron recogidas a través de un grabador portátil Sanyo M-1111. Las tareas que se utilizaron para su elicitación (ver próximo apartado) emplearon como material el siguiente:

- Una película de videotape, realizada para esta investigación por el profesor Fernando Campos (Facultad de Ciencias de la Información, U.C.M.) sobre la base del guión utilizado por Chaika y Alexander en sus invèstigaciones 
- Dos láminas no ambiguas de contenido emocionalmente neutro. La primera de ellas, en blanco y negro $(43 \times 30 \mathrm{~cm}$.), representa una escena simple con sólo nueve unidades figurativas, y fue diseñada para este estudio por un dibujante profesional (Miguel Angel Herranz). La segunda, a color $(35 \times 27 \mathrm{~cm}$.), y más compleja, representa una escena campestre infantil. Esta lámina forma parte del material original de la prueba T.E.D.E.P.E., diseñada en 1984 por Rivière, Belinchón y otros, para la evaluación de las alteraciones del desarrollo en edad preescolar (actualmente, en proceso de baremación).

- Una adaptación de la «Historia del corsario Landolfo Rúfolo» del Libro Primero del Decamerón de Boccaccio, realizada por la autora de este trabajo sobre la base de los criterios definidos por Van Dijk en 1979.

La evaluación a través del WAIS, la administración del C.P.T. y la recogida de las muestras lingüísticas fue realizada, íntegramente, por la autora de este trabajo (M.B.).

\section{Tareas e índices lingüísticos diseñados para el estudio}

El proceso de elaboración y diseño de las tareas e índices sobre los que se realizó la comparación de las muestras lingüísticas de los dos grupos de nuestro estudio tomó como punto de referencia teórico, como ya señalamos, algunos de los conceptos y los métodos desarrollados por los psicólogos del lenguaje que, desde una orientación textual y/o cognitiva, han tratado de ofrecer hipótesis comprensivas de la producción de los discursos en situaciones naturales de interacción comunicativa en los últimos años (cfr. revisiones en Mayor, 1984; Carroll, 1986; Belinchón, en prensa). La adopción de este enfoque se justificó tanto por la identificación que tradicionalmente se ha hecho del término «lenguaje esquizofrénico» con la noción de «discurso desviado» como por los resultados obtenidos en algunas de las investigaciones más recientes sobre este tipo de lenguaje desde esta misma perspectiva teórica (vg. Rochester y Martin, 1979; Hoffman et al., 1982, y en prensa, Andreasen et al., 1985; Chaika y Alexander, 1982; cfr. Belinchón, 1987, y en este mismo volumen).

Cuatro tareas de producción verbal y sesenta y ocho índices de medida o evaluación lingüística fueron diseñados para la recogida y análisis de los textos del estudio, sobre la base de tres supuestos que, hoy por hoy, podrían considerarse básicos dentro del enfoque textual:

1) Los discursos son unidades de lenguaje en uso (unidades funcionales) que poseen regularidades estructurales cuya «bondad» o «desviación» debe ser definida en función, entre otras cosas, del contexto comunicativo en que se producen y las demandas informativas impuestas por la tarea (Clark y Clark, 1978; Van Dijk, 1980a, 1980b, 1984, 1985).

2) La producción textual constituye un proceso de composición complejo que requiere, cuando menos, cuatro grandes fases: la construcción de un primer esquema proposicional, la organización de este esquema en unidades semánticas más específicas, la codificación de formas lingüísticas coherentes en la estructura superficial y la producción fonoarticulatoria (Scinto, 1977, 1982; Kintsch y Van Dijk, 1978; Van Dijk, 1980a; Zammuner, 1981).

3) La impresión de coherencia de los discursos o textos puede ser descrita empíricamente tanto a partir de variables «centradas en la organización del texto» como a partir de variables «orientadas a la mente tanto del hablante como del oyente» (cfr. Scinto, 1977, 1984; De Beaugrande, 1980; Belinchón, en este mismo volumen).

Las tareas utilizadas en este estudio fueron cuatro: 1) la narración de una película breve ( $4 \mathrm{~min}$.) y emocionalmente neutra recién presentada en video; 2) la explicación, por parte de los sujetos, de su opinión a favor o en contra 
de tres tópicos de actualidad en el momento de administrar las pruebas - de los que se seleccionó uno solo (la opinión sobre el ingreso de España en la OTAN)-; 3) la descripción de dos láminas de temática emocionalmente neutra, y 4) la narración de un relato breve previamente leído por el examinador (concretamente, la «historia del corsario Landolfo Rúfolo» de Boccaccio).

Estas cuatro tareas se solicitaban al sujeto a lo largo de una sesión ininterrumpida de conversación (de entre treinta y cuarenta y cinco minutos de duración) sostenida con la autora de este trabajo. Aunque en la sesión de prueba, las cuatro tareas se incluían en el marco de una misma entrevista, su configuración (es decir, ir precedidas de la presentación de un material consigna específico en cada caso) permite considerarlas como tareas independientes, de tipo monológico, bien definidas en cuanto a sus referentes y capaces de proporcionar información respecto al grado de ajuste contextual de los discursos de los sujetos a las demandas impuestas por el contexto.

Vistas desde la perspectiva del tipo de respuestas que pueden elicitar, las cuatro tareas pueden interpretarse como elicitadoras de discursos que requieren una comunicación propositiva y que admiten una longitud y estructuración variable por parte de los sujetos. Desde ese punto de vista, por tanto, aparecen como idóneas para la valoración de dimensiones como la productividad, la organización sintáctica y macroestructural, la ambigüedad referencial y la cohesión que, en diferentes estudios e investigaciones anteriores, han permitido diferenciar empiricamente las producciones de sujetos esquizofrénicos y normales (cfr. Belinchón, en este mismo volumen).

Las cuatro tareas diseñadas cuentan con precedentes empíricos directos tanto en la investigación psicolingüística básica como en el contexto de la investigación del lenguaje esquizofrénico. La tarea de narración de películas fue utilizada por primera vez con esquizofrénicos por Chaika y Alexander (1982) y, en el contexto de la investigación básica, ha sido estudiada también por Baggett (1979) y, sobre todo, por Chafe (1980). La tarea de explicación se reveló como metodológicamente interesante para el estudio del lenguaje esquizofrénico en los trabajos de Rochester et al. (1977) y Rochester y Martin (1979). Las descripciones de láminas o escenas no ambiguas, además de ser empleada también por Rochester y su equipo, constituyó el objetivo central del trabajo de Reich y Cutting (1982). Respecto a la narración de historias o relatos breves, los trabajos de Van Dijk y, muy especialmente, la serie de experimentos que publicó en 1979, constituyeron el punto de referencia fundamental para su inclusión y análisis en este estudio. En el estudio de Rochester y Martin (1979), este tipo de tarea (con una duración y contenido distintos) fue también utilizada con sujetos esquizofrénicos y controles.

Los índices lingüísticos, por su parte, resultaron de una operativización de la noción de «coherencia» que (como se sugirió en un trabajo anterior - Belinchón, en este mismo volumen-) la pone en relación con siete dimensiones de análisis que pueden considerarse como clásicas en el estudio psicológico de la producción del lenguaje esquizofrénico: la fluidez verbal, la comisión de errores gramaticales, la adecuada o inadecuada organización de vínculos cohesivos e indicadores referenciales no ambiguos, la coherencia proposicional y la organización macroestructural de los discursos.

Además de estos índices se evaluó también la longitud de las emisiones, al objeto de verificar algunas de las observaciones empíricas anteriores (vg. Rochester y Martin, 1979; Allen, 1983) relativas a una menor longitud de las producciones de los esquizofrénicos frente a la de sus controles normales.

Esquemáticamente, los índices utilizados en el estudio y las fuentes más 166 directas en las que se basó su operativización aparecen recogidos en las tablas IV a XIV. 
N. ${ }^{9}$ total de palabras/tarea.

N.. total de frases/tarea.

N. 9 total de oraciones/tarea.

N. ${ }^{9}$ de palabras/frase bien construida.

N.@ de palabras/oración.

N. 9 de frases/oración.

TABLA V

Indices utilizados en la valoración de los errores de fluidez

\begin{tabular}{|c|c|c|}
\hline Categoria & Criterio & Tipos \\
\hline Titubeo. & $\begin{array}{l}\text { Producción de uno o varios so- } \\
\text { nidos que no guardan ninguna } \\
\text { relación estructural con la deci- } \\
\text { sión léxica subsiguiente. } \\
\text { (p. ej. «mm»). } \\
\text { Repetición, sin modificaciones, } \\
\text { de una o varias palabras antes } \\
\text { de concluir la frase o constitu- } \\
\text { yente gramatical. } \\
\text { (p. ej. «entonces vino vino el } \\
\text { padre y le dio el dinero para el } \\
\text { helado). } \\
\text { Utilización de «muletillas». } \\
\text { (p. ej, «o sea», «esto», etc.). }\end{array}$ & \\
\hline
\end{tabular}

Falsos inicios. Inicio, interrupción y elaboración posterior de una unidad lingüística.
FI. 1: Afecta sólo al primer fonema de la palabra.

FI. 2: Afecta sólo a una palabra (p. ej. «empieza a ata a atrac o sea a atracar barcos»).

FI. 3: Afecta a la producción de un constituyente gramatical (p. ej. «entonces pues se compra marcan, se compra determinadas mercancías»).

FI. 4: Afecta al completamiento de la frase (p. ej. «pues decían que Landulfo se había era un comerciante italiano").

Repeticiones. Repetición sin modificaciones de palabras, constituyentes o frases después de haberse completado la producción del constituyente, frase u oración.
R 1: Repetición de palabras (ej. «entonces se compró compró un helado»).

R 2: Repetición de constituyentes (ej. "después se ve una mujer con un niño pequeño en brazos con un niño pequeño en brazos").

R 3: Repetición de frases (ej. «luego llega el padre y le da dinero para comprar el helado, llega el padre y le da dinero para com. prar el heladom). 


\begin{tabular}{cl}
\hline Categoría & \multicolumn{1}{c}{ Criterio } \\
\hline Proposiciones incompletas & Oraciones y/o frases gramaticales que contienen \\
& el verbo, pero carecen total o parcialmente de \\
& constituyentes que son básicos para la interpre- \\
& tación de su significado (p. ej. «y entonces el pa- \\
& dre se le ha quedao, bueno no quería darle el di- \\
& nero»).
\end{tabular}

Error de conexión

Utilización inadecuada de los conectivos gramaticales (ej. «luego a la chica éste le ha entrao envidia como la otra pedía un polo no?»)

Error de concordancia

Mala concordancia en la construcción de los morfemas de género, número, persona o tiempo verbal (p. ej. «primero se ve imágenes de una calle por la mañana»).

Errores en la construcción gramatical de la proposición principal de la oración.
Cualquiera de los anteriores en la proposición principal de la oración.

TABLA VII

Indices de organización y complejidad sintáctica utilizados en el presente estudio

Porcentaje de oraciones simples (sobre total de oraciones).

Porcentaje de oraciones compuestas (sobre total de oraciones).

Porcentaje de frases sin nexo gramatical - proposiciones yuxtapuestas e incrustadas- (sobre total de frases).

Porcentaje de proposiciones coordinadas (sobre total de frases).

Porcentaje de proposiciones subordinadas (sobre total de frases).

Porcentaje de proposiciones gramaticales bien construidas (sobre total de proposiciones) -es decir, proposiciones completas y sin error gramatical-.

\section{TABLA VIII}

Categorias utilizadas en el estudio para el análisis de los recursos de cobesión

\begin{tabular}{ll}
\hline Tipo & Subtipo \\
\hline 1) Vínculos de cohesión referencial. & R 1: Referencia personal. \\
& R 2: Demostrativos, posesivos y artículos \\
& R 3: Comparatos. \\
& C 1: Conjunción aditiva. \\
2) Vínculos de conexión gramatical. & C 2: Conjunción adversativa. \\
& C 3: Conjunción causal. \\
& C 4: Conjunción temporal-espacial. \\
& L 1: Item idéntico o de igual raíz. \\
3) Vínculos de cohesión léxica. & L S: Sinónimo o casi sinónimo. \\
& L 3: Item superordinado. \\
& L 4: Item general.
\end{tabular}


TABLA IX

Categorías utilizadas en la valoración de las estrategias de indicación referencial de los grupos fóricos (operativización adaptada de Martin, 1974)

\begin{tabular}{ll}
\hline Tipo & Subtipo \\
\hline Endófora. & Anáfora. \\
& Catáfora. \\
Exófora. & \\
Referente implícito. & \\
Referente ambiguo o déficit de determinación. & \\
\hline
\end{tabular}

TABLA X

Taxonomia de relaciones proposicionales (en el plano de la coberencia lineal) utilizados en el estudio

\begin{tabular}{ll}
\hline De tipo condicional/temporal & De tipo funcional \\
\hline Causas/razones. & Especificación. \\
Componentes. & Generalización. \\
Consecuencias/objetivos. & Explicación. \\
Ocasión. & Contraste. \\
Permisión. & Ejemplo. \\
Se suceden en el tiempo. & Paralelo. \\
Se dan simultáneamente. & Corrección. \\
& Preparación. \\
\hline
\end{tabular}

\section{TABLA XI}

Categorias utilizadas en este estudio para la valoración de la coherencia de los discursos

Porcentaje de proposiciones con vínculos proposicionales explícitos y correctos (en su formulación superficial textual).

Porcentaje de proposiciones implícitas que deberían ser necesariamente explícitas (es decir, cuya vinculación lógica o pragmática con las otras proposiciones del discurso resulta difícil de establecer sobre la base del texto dado).

Porcentaje de proposiciones cuya vinculación está incorrectamente establecida.

Porcentaje de proposiciones «extradiscursivas» (es decir, que sólo tangencialmente están relacionadas con el tópico del discurso). 
Dimensiones de valoración macroestructural para los textos narrativos

\begin{tabular}{|c|c|c|}
\hline Dimensión & Indices & Criterio \\
\hline a.1. & $\begin{array}{l}\text { Porcentaje de constituyen- } \\
\text { tes «completos». } \\
\text { Porcentaje de constituyen- } \\
\text { tes «incompletos». } \\
\text { Porcentaje de constituyen- } \\
\text { tes «insuficientes». } \\
\text { Porcentaje de adiciones y } \\
\text { distorsiones. }\end{array}$ & $\begin{array}{l}\text { Incluye la información argumental- } \\
\text { mente básica y al menos parte de la in- } \\
\text { formación secundaria que correspon- } \\
\text { de a tal constituyente (ver nota). } \\
\text { Sólo contiene el elemento nuclear de } \\
\text { la información. } \\
\text { Se incluye únicamente información ar- } \\
\text { gumentalmente necesaria. } \\
\text { Contiene errores o información argu- } \\
\text { mentalmente irrelevante. }\end{array}$ \\
\hline a. 2 . & $\begin{array}{l}\text { Porcentaje de errores de or- } \\
\text { den intraconstituyentes. } \\
\text { Porcentaje de errores de or- } \\
\text { den interconstituyentes. }\end{array}$ & $\begin{array}{l}\text { Afectan a la ordenación de los even- } \\
\text { tos dentro del propio constituyente. } \\
\text { Afecta a la ubicación del constituyen- } \\
\text { te en la secuencia total del discurso. }\end{array}$ \\
\hline a.3. & $\begin{array}{l}\text { Porcentaje de conexión ade- } \\
\text { cuada-explícita. } \\
\text { Porcentaje de conexión ade- } \\
\text { cuada-implícita. } \\
\text { Porcentaje de conexión ina- } \\
\text { decuada. }\end{array}$ & $\begin{array}{l}\text { El hablante vincula temática o tempo- } \\
\text { ralmente el contenido de los constitu- } \\
\text { yentes. } \\
\text { El hablante superpone los constitu- } \\
\text { yentes en un orden correcto, pero sin } \\
\text { vincular explícitamente su significado. } \\
\text { El hablante distorsiona las relaciones } \\
\text { de conexión y/o el orden de los cons- } \\
\text { tituyentes. }\end{array}$ \\
\hline a.4. & $\begin{array}{l}\text { N. }{ }^{0} \text { de constituyentes tex- } \\
\text { tuales que componen la na- } \\
\text { rración. }\end{array}$ & $\begin{array}{l}\text { Se evalúa por comparación con el nú- } \\
\text { mero de constituyentes de que se com- } \\
\text { ponía el material original (en nuestro } \\
\text { caso, la película y la historia). } \\
\text { (Cfr. nota.). }\end{array}$ \\
\hline
\end{tabular}

TABLA XIII

Dimensiones de valoración macroestructural para el análisis de los textos explicativos

\begin{tabular}{|c|c|c|}
\hline Dimensión & Indices & Criterio \\
\hline b.1. & $\begin{array}{l}\text { Porcentaje de premisas ob- } \\
\text { jetivas. } \\
\text { Porcentaje de premisas sub- } \\
\text { jetivas } \\
\text { Porcentaje de premisas } \\
\text { mixtas. }\end{array}$ & $\begin{array}{l}\text { Se basa en criterios de autoridad ex- } \\
\text { ternos al sujeto. } \\
\text { Se basa en opiniones, valoraciones } \\
\text { personales o criterios de autoridad no } \\
\text { demostrables objetivamente. } \\
\text { Combina las dos anteriores. }\end{array}$ \\
\hline b. 2 . & $\begin{array}{l}\text { Porcentaje de premisas con } \\
\text { desarrollo argumental «0». } \\
\text { Porcentaje de premisas con } \\
\text { desarrollo argumental «1». } \\
\text { Porcentaje de premisas con } \\
\text { desarrollo argumental « } 2 » .\end{array}$ & $\begin{array}{l}\text { Contienen únicamente el enunciado } \\
\text { de la premisa. } \\
\text { Contienen al menos una razón de apo- } \\
\text { yo a la premisa, pero no se desarrolla: } \\
\text { simplemente se enuncia. } \\
\text { La razón que apoya o justifica las pre- } \\
\text { misas son explicadas a su vez por el } \\
\text { sujeto. }\end{array}$ \\
\hline
\end{tabular}


Indices de valoración macroestructural utilizados para el análisis de los textos en la tarea de "descripción"

\begin{tabular}{|c|c|c|}
\hline Dimensión & Indices & Criterio \\
\hline \multirow[t]{3}{*}{ c.1. } & $\begin{array}{l}\text { Porcentaje constituyentes } \\
\text { completos. }\end{array}$ & $\begin{array}{l}\text { Incluyen la descripción de los elemen- } \\
\text { tos o rasgos temáticamente más rele- } \\
\text { vantes de las unidades funcionales de } \\
\text { la escena al menos y parte de los se- } \\
\text { cundarios (ver nota). }\end{array}$ \\
\hline & $\begin{array}{l}\text { Porcentaje constituyentes } \\
\text { incompletos. } \\
\text { Porcentaje constituyentes } \\
\text { insuficiente. }\end{array}$ & $\begin{array}{l}\text { Preserva el rasgo temático nuclear, sin } \\
\text { incluir ninguna información más. } \\
\text { La descripción se centra tan sólo en } \\
\text { detalles secundarios de las unidades de } \\
\text { la escena, omitiendo el rasgo temáti- } \\
\text { camente nuclear. }\end{array}$ \\
\hline & $\begin{array}{l}\text { Porcentaje adiciones, erro- } \\
\text { res o distorsiones. }\end{array}$ & $\begin{array}{l}\text { Añade información no presente en la } \\
\text { escena original y/o interpretaciones } \\
\text { incorrectas de las mismas. }\end{array}$ \\
\hline \multirow[t]{2}{*}{ c. 2 . } & Puntuación «l». & $\begin{array}{l}\text { La definición explícita del «tema» se } \\
\text { ubica en el inicio o el final de la des- } \\
\text { cripción. }\end{array}$ \\
\hline & Puntuación «2». & $\begin{array}{l}\text { La descripción de la escena no contie- } \\
\text { ne ninguna definición explícita del } \\
\text { «tema» o bien éste se presenta de for- } \\
\text { ma fragmentada o incompleta a lo lar- } \\
\text { go del texto. }\end{array}$ \\
\hline
\end{tabular}

\section{Procedimiento general de obtención de los datos del estudio}

La estructuración de las sesiones de prueba en que se realizaba la evaluación intelectual de los sujetos, se recogían las muestras de producción verbal y se administraba la tarea atencional fue idéntica para todos los sujetos.

Una vez el sujeto en la sala de prueba, el examinador explicaba los objetivos de la investigación y, a continuación, iniciaba con el sujeto una conversación distendida y no directiva acerca de los motivos de ingreso del paciente en el hospital (en el grupo experimental) y/o de su ocupación habitual, nivel de estudios, situación familiar, etc. Tras este primer período (de 5 a 10 minutos de duración, no incluido entre las muestras lingüísticas a analizar), se presentaban sucesivamente las cuatro tareas lingüísticas, encadenadas entre sí por comentarios de carácter general no relevantes para el análisis.

Una vez finalizada la cuarta tarea (narración de la historia), el sujeto tenía un período de descanso (cuya duración él mismo determinaba) y, posteriormente, realizaba la tarea del C.P.T. y las tareas del WAIS. En el caso de los pacientes esquizofrénicos, fue preciso, con frecuencia, articular un nuevo período de descanso durante la realización de estas últimas tareas.

La evaluación clínica de los pacientes y de los sujetos controles fue realizada en sesiones distintas por el psiquiatra encargado de la misma (Dr. Jorge Grijalbo).

\section{Transcripción y preparación de las muestras lingüísticas}

Las grabaciones de cada sujeto (30-40 min.) fueron transcritas íntegramente a papel (ver nota 1). Sobre la base de esta transcripción (literal pero 
no fonética) se seleccionaron los textos correspondientes a las cuatro tareas (narración de la película, explicación de opiniones, descripción de las láminas y narración de la historia de Boccaccio). Los textos fueron mecanografiados en su totalidad y preparados en hojas independientes para su análisis posterior (en estas hojas, el nombre de los sujetos fue sustituido por un número asignado aleatoriamente para garantizar la objetividad del proceso de codificación).

\section{Fiabilidad de los índices}

Para el cálculo de la fiabilidad de las categorías lingüísticas, se seleccionó un conjunto de 40 protocolos (25 por 100 del total), en los que estaban proporcionalmente representados los cuatro tipos de discurso y los dos grupos de sujetos.

En el proceso participó un total de cinco personas (cuatro estudiantes de quinto curso de Psicología de la U.A.M. y la autora de este trabajo, M.B.). Cada dimensión del análisis fue codificada por dos personas (un estudiante y M.B.), si bien el análisis estadístico de los resultados se basó en la codificación realizada por esta última. En todos los casos, el análisis de los protocolos para el cálculo de la fiabilidad fue realizado tras una serie de ensayos y reuniones destinadas a la familiarización de los observadores con las dimensiones teóricas y empíricas del estudio. Los textos seleccionados para este análisis procedían de sujetos experimentales y controles que, tras haber realizado las pruebas, fueron excluidos de las muestras finales de la investigación.

\section{TABLA XV}

Proporción media de acuerdos entre observadores en el cálculo de fiabilidad de las categorias del estudio

\begin{tabular}{|c|c|}
\hline Dimensión de análisis & $\begin{array}{c}\text { Porcentaje de acuerdos entre } \\
\text { observadores }\end{array}$ \\
\hline Productividad. & 99,8 \\
\hline Errores de fluidez. & 91,3 \\
\hline Errores gramaticales. & 96,00 \\
\hline Porcentaje frases bien construidas. & 80,98 \\
\hline Tipo de oración. & 87,4 \\
\hline Vínculos de cohesión ( $\overline{\mathrm{X}}$ total). & 86 \\
\hline Distancia vínculos cohesión. & 81,2 \\
\hline Foricidad. & 79,6 \\
\hline Coherencia ( $\overline{\mathrm{X}}$ total). & 76,15 \\
\hline Porcentaje vínculos esplícitos. & 87,3 \\
\hline Porcentaje vínculos necesariamente explícitos. & 68,1 \\
\hline Porcentaje vínculos no coherentes. & 76 \\
\hline Porcentaje proposiciones extradiscursivas. & 73,2 \\
\hline Calidad representacional módulos. & 71,43 \\
\hline Tipo de conexión entre módulos. & 83,33 \\
\hline Errores de ordenación de los módulos. & 100 \\
\hline
\end{tabular}

tal y como se recoge en la Tabla XV, el grado de acuerdo entre los observadores resultó ser aceptablemente bueno para todas las dimensiones analizadas si bien, globalmente, resultó algo más bajo que los índices de fiabilidad obtenidos por otros autores (ver tabla XVI).

La diferencia en el nivel de fiabilidad de nuestro estudio no parece afectar, sin embargo, a la tendencia general observada en otros trabajos: confir- 


\begin{tabular}{lcccc}
\hline & Productividad & Cohesión & Foricidad & Coherencia \\
\hline Rochester y Martin (1979). & - & $92,7 \%$ & $91 \%$ & - \\
Fine y Bartolucci (1981). & - & $91,5 \%$ & $75 \%$ & - \\
Harvey (1983). & $95 \%$ & $86,67 \%$ & $82,5 \%$ & - \\
Hoffman et al. (1982). & - & - & - & $96 \%$ \\
\hline
\end{tabular}

ma la mayor facilidad para obtener índices altos en fiabilidad con dimensiones lingüísticas "centradas en el texto» (como en el caso de la "productividad verbal», la «gramaticalidad de las oraciones», etc.), y la dificultad (o, si se prefiere, la mayor variabilidad interjueces) cuando las dimensiones tienen un fuerte contenido semántico y pragmático (vg. la «coherencia proposicional»).

Desde un punto de vista teórico, los problemas de fiabilidad de este último conjunto de índices resultan explicables si asumimos que la comprensión (y/o la valoración de un discurso) es, fundamentalmente, un proceso de «reconstrucción» que exige del oyente presuposiciones y competencias extraordinariamente complejas (cfr. Van Dijk, 1985). Empíricamente, Fine y Bartolucci (1981) han destacado las variaciones contextuales y del nivel de desorganización de los discursos como factores que pueden reducir los valores de fiabilidad de los índices lingüísticos aun en el caso de observadores bien entrenados. Esta observación y el hecho de que, hasta la fecha, no conocemos otros estudios que hayan utilizado este tipo de índices en el análisis de muestras lingüísticas en castellano, nos permite suponer, en principio, que nuestro trabajo, a pesar de su carácter preliminar, reúne las condiciones mínimas de fiabilidad que son exigibles a cualquier investigación científica.

\section{RESULTADOS}

\section{Significación diagnóstica de los índices lingüísticos utilizados en el estudio}

Con el fin de comprobar la viabilidad de una diferenciación entre sujetos esquizofrénicos y normales sobre la base exclusiva del análisis lingüístico de sus producciones verbales, se realizaron dos análisis estadísticos complementarios que, presumiblemente, podian proporcionar evidencia convergente.

El primero de ellos fue un análisis de varianza con un covariado con medidas repetidas en un factor (programa $2 \mathrm{~V}$ del paquete estadístico BMDP). Con dicho análisis, tratamos de comprobar si, anulado el efecto de la variable C.I., era posible encontrar diferencias entre los esquizofrénicos y los normales en las variables e indices lingüísticos diseñados en este estudio. El segundo, fue un análisis discriminante (BMDP7M) del que esperábamos obtener el conjunto de variables lingǘsticas capaz de proporcionar la máxima diferenciación entre los dos grupos de sujetos de nuestro estudio, así como una clasificación de los sujetos en sus respectivos grupos diagnósticos que contuviera menos errores que los esperables de una clasificación al azar.

Los resultados obrenidos en el análisis de covarianza fueron, tomados a grandes rasgos, consistentes con las hipótesis de partida. Tras controlar estadísticamente el efecto de la variable C.I., el análisis del factor «grupo» sobre el conjunto total de variables dependientes analizadas permitió identificar un 
conjunto de 17 variables lingüísticas, capaces de diferenciar entre los pacientes y los controles con una probabilidad de error inferior al 5 por 100. De estas 17 variables, 1 correspondía al nivel de «fluidez verbal», 1 al de «gramaticalidad de las operaciones», 3 al plano de la cohesión sintáctica y semántica de la estructura superficial del texto, 1 al plano de los indicadores referenciales, 4 al plano de la coherencia proposicional y 7 al plano de los indicadores de la organización macroestructural de los discursos (ver tabla XVII). Un análisis en profundidad de los efectos de la variable «tarea» y la interacción "grupo × tarea» está siendo realizado todavía en el momento de redactar estas líneas (cfr. Belinchón, en preparación).

\section{TABLA XVII}

Resultados significativos (probabilidad de error menor que el 5 por 100) del análisis de covarianza

\begin{tabular}{|c|c|c|c|c|c|c|}
\hline \multirow{2}{*}{ Variable } & \multicolumn{2}{|c|}{ Esquizofrenia } & \multicolumn{2}{|c|}{ Normales } & \multirow{2}{*}{$\mathbf{F}$} & \multirow{2}{*}{$\begin{array}{l}\text { Nivel } \\
\text { signif. }\end{array}$} \\
\hline & $\mathbf{x}$ & DT & $\mathbf{x}$ & DT & & \\
\hline Porcentaje de titubeos. & 61,34 & 21,8 & 73,57 & 19,73 & 6,71 & $\mathrm{p}<.02$ \\
\hline $\begin{array}{l}\text { Porcentaje de frases bien } \\
\text { construidas. } \\
\text { Promedio de vínculos deco- }\end{array}$ & 83,46 & 6,25 & 89,30 & 4,57 & 6,74 & $\mathrm{p}<.02$ \\
\hline hesión por oración. & 3,00 & 0,8 & 4,02 & 0,98 & 5,86 & $\mathrm{p}<.05$ \\
\hline $\begin{array}{l}\text { Promedio de vínculos de re- } \\
\text { ferencia por oración. } \\
\text { Promedio de distancia de }\end{array}$ & 19,60 & 5,17 & 26,05 & 7,56 & 8,08 & $\mathrm{p}<.01$ \\
\hline $\begin{array}{l}\text { vínculos de cohesión. } \\
\text { Proporción de referentes }\end{array}$ & 3,24 & 0,63 & 2,79 & 0,56 & 4,97 & $\mathrm{p}<.05$ \\
\hline $\begin{array}{l}\text { ambiguos en los GNF. } \\
\text { Porcentaje de proposicio- }\end{array}$ & 9,70 & 7,48 & 5,01 & 3,91 & 4,33 & $\mathrm{p}<.05$ \\
\hline & 71,46 & 12,2 & 85,19 & 8,94 & 7,69 & $\mathrm{p}<.01$ \\
\hline $\begin{array}{l}\text { nes de vínculos necesaria- } \\
\text { mente explícitos. } \\
\text { Porcentaje de proposicio- }\end{array}$ & 7,81 & 4,48 & 2,19 & 2,57 & 13,81 & $\mathrm{p}<.001$ \\
\hline $\begin{array}{l}\text { nes de vínculos no coheren- } \\
\text { tes. }\end{array}$ & 6,27 & 6,32 & 0,88 & 1,89 & 7,67 & $\mathrm{p}<.01$ \\
\hline $\begin{array}{l}\text { Porcentaje } \\
\text { NE+NC+EXTRAD. } \\
\text { Porcentaje de módulos III }\end{array}$ & 21,21 & 12,97 & 6,81 & 6,47 & 10,79 & $\mathrm{p}<.01$ \\
\hline $\begin{array}{l}\text { (total). } \\
\text { Porcentaje de módulos VI }\end{array}$ & 35,02 & 14,68 & 20,02 & 16,48 & 4,21 & $\mathrm{p}<.05$ \\
\hline $\begin{array}{l}\text { (total). } \\
\text { Porcentaje de módulos VI }\end{array}$ & 26,61 & 15,91 & 10,32 & 7,66 & 10,65 & $\mathrm{p}<.01$ \\
\hline $\begin{array}{l}\text { (narrac.). } \\
\text { Porcentaje de módulos I y }\end{array}$ & 37,81 & 22,86 & 13,65 & 11,77 & 13,02 & $\mathrm{p}<.001$ \\
\hline $\begin{array}{l}\text { II (total). } \\
\text { Porcentaje de módulos I y }\end{array}$ & 61,15 & 17,08 & 79,20 & 16,28 & 5,77 & $\mathrm{p}<.05$ \\
\hline $\begin{array}{l}\text { Il (narrac.). } \\
\text { Congruencia con el material }\end{array}$ & 61,57 & 22,78 & 89,24 & 14,3 & 11,52 & $\mathrm{p}<.01$ \\
\hline $\begin{array}{l}\text { original (narraciones). } \\
\text { Congruencia con el material }\end{array}$ & 79,63 & 18,25 & 97,71 & 6,82 & 10,15 & $\mathrm{p}<.01$ \\
\hline original (historia). & 72,22 & 29,87 & 98,75 & 5,59 & 9,17 & $\mathrm{p}<.01$ \\
\hline
\end{tabular}

El análisis discriminante fue realizado sobre un total de 19 variables lingüisticas seleccionadas sobre la base de los criterios propuestos por Klecka (1984) - ver tabla XVIII-.

Los resultados que proporcionó este análisis fueron, también, claramente congruentes con nuestras hipótesis de partida y con los resultados obtenidos 
Total de palabras por tarea.

Total de palabras por oración.

Promedio de errores fluidez por oración.

Promedio de errores gramaticales por oración.

Promedio de frases bien construidas.

Proporción de oraciones simples.

Promedio de vínculos contenidos por oración.

Promedio de la distancia de vínculos de cohesión.

Proporción de endóforas.

Proporción de endóforas + GNF con referente implícito.

Proporción de GNF con referente ambiguo.

Proporción de proposiciones con vínculo explícito.

Proporción de proposiciones NE + NC + EXTRADISCURSIVAS.

Proporción de módulos representacionalmente insuficientes.

Proporción de módulos con adiciones irrelevantes o erróneas.

Proporción de módulos completos + incompletos.

Proporción de constituyentes textuales mal ordenados.

Nivel de desarrollo argumental.

Congruencia temática con el original (en narraciones).

en estudios anteriores. Se obtuvo una función discriminante integrada por dos variables de fuerte «peso» semántico («la distancia entre los vínculos de cohesión y sus referentes» y la suma de «proposiciones necesariamente explícitas, no coherentes y extradiscursivas»), capaz de explicar el 35,75 por 100 de la varianza total y de clasificar correctamente en sus grupos al 82,4 por 100 del total de los sujetos del estudio $(64,7$ por 100 de los esquizofrénicos y 100 por 100 de los normales).

Sobre la base de las mismas variables lingüísticas, un análisis discriminante posterior, que utilizó como base para la clasificación de los sujetos la dimensión clínica de «desorden formal de pensamiento» en vez de la de «esquizofrenia» no permitió clasificar correctamente en sus respectivos grupos diagnósticos más que al 10 por 100 de los esquizofrénicos con DFP, el 14,3 por 100 de los esquizofrénicos sin DFP y el 94,1 por 100 de los sujetos normales.

Así, pues, y globalmente considerados, los resultados obtenidos a través de estos dos tipos de análisis confirmaron la hipótesis general de que a partir del análisis de los discursos verbales es posible identificar o definir marcadores lingüísticos capaces de diferenciar entre sujetos esquizofrénicos y normales. Asimismo, confirmaron la hipótesis de que esta diferenciación es viable aunque el grupo de pacientes sea clínicamente heterogéneo (entre otras, respecto a la variable clínica de DFP) y aunque no se realice una selección previa de las muestras lingüísticas a partir de criterios clínicos de desviación.

Desde un punto de vista teórico, los resultados obtenidos presentan puntos de extraordinaria coincidencia e interés respecto a algunas de las investigaciones anteriores.

En primer lugar, nuestros datos confirman la hipótesis (defendida en los últimos años sobre todo por Chaika 1974, 1977, 1982a, 1982 b; Rochester y Martin, 1979; Hoffman et al., 1982; y Fraser et al., 1986) de que, comparados con un grupo de sujetos formales, los esquizofrénicos presentan una perturbación en su capacidad para producir discursos estructuralmente bien construidos. Comparados con los de sus controles, los discursos de nuestros pacientes presentaron, por ejemplo, los siguientes rasgos: 
- ser menos fluidos y estar sintácticamente peor construidos $(\mathrm{F}(1,38)=6,71 ; \mathrm{p}<.02$, en "proporción de titubeos por frase»; $\mathrm{F}(1,36)=6,74$; $\mathrm{p}<.02$ en "proporción de frases bien construidas»);

- ser menos coherentes tanto en su estructura superficial como a nivel proposicional $(\mathrm{F}(1,36)=5,86 ; \mathrm{p}<.05$ en «promedio de vínculos de cohesión por oración»; $F(1,38)=7,69 ; \mathrm{p}<.01$ en «proporción de vínculos proposicionales explícitos y adecuados»; $F(1,30)=13,81 ; \mathrm{p}<.001$ en «proposiciones necesariamente explícitas ausentes del texto»; $(\mathrm{F}(1,30)=7,67 ; \mathrm{p}<.01$ en «vínculos no lógicos de coherencia»), y

- estar peor estructurados semánticamente en el nivel de los constituyentes macrotextuales $(\mathrm{F}(1,36)=5,77 ; \mathrm{p}<.05$ en «módulos representacionales completos»; $\mathrm{F}(1,36)=4,21 ; \mathrm{p}<.05$ en «módulos representacionalmente insuficientes»; $F(1,24)=10,6 ; p<.01$ en «módulos con adiciones extradiscursivas") ).

Tomados en su conjunto, estos rasgos sugieren la existencia de déficit relacionados con la planificación semántica global de los discursos (es decir, la llamada «macroestructura textual») y/o con la producción de formas lingüísticas coherentes capaces de «realizar» o de «actualizar» el significado de la «macroestructura» en el nivel de la estructura superficial del discurso. Empíricamente, estos déficit parecen poder ser identificados tanto a partir de análisis oracionales individuales como a partir de análisis lingüísticos de carácter suprafrasal.

En segundo lugar, nuestros datos confirman la hipótesis de que existe un déficit comunicativo, en los esquizofrénicos, que afecta claramente (aunque no de forma exclusiva), a los aspectos referenciales y pragmáticos de los discursos.

Desde un punto de vista referencial, por ejemplo, los discursos de los esquizofrénicos revelaron plantear más dificultades de interpretación que los de sus controles normales. Tal dificultad se derivaria, fundamentalmente, de:

- la utilización más frecuente de grupos nominales con referentes ambiguos $(\mathrm{F}(1,36)=4,33 ; \mathrm{p}<.05)$,

- la mayor distancia textual entre los vínculos cohesivos y sus referentes $(\mathrm{F}(1,35)=4,97 ; \mathrm{p}<.05)$ y

- la mayor borrosidad referencial tanto de los grupos nominales fóricos como de la organización semántica global del discurso $(\mathrm{F}(1,21)=10,15 ; \mathrm{p}<.01$ en «congruencia del discurso respecto a su referente original en la narración de la película; $(F(1,21)=9,17 ; p<.01$ en «congruencia con el material original en la tarea de narración de la historia»).

Los datos anteriormente expresados permiten sospechar, pues, que este tipo de pacientes carece de una "teoría de la mente» de sus interlocutores eficaz para predecir la conducta del oyente y/o para inferir correctamente sus estados de conocimiento durante el proceso de la interacción verbal. La metáfora de la teoría de la mente, utilizada inicialmente por Baron-Cohen, Leslie y Frith en 1985 para caracterizar los déficit cognitivos y comunicativos de los niños autistas, no ha sido explorada de forma directa con los pacientes esquizofrénicos, sin embargo, por su similitud con la «hipótesis del auditor imaginario» propuesta por Sullivan (1925) - y verificada experimentalmente, años después, por Cohen (1978), Rochester et al. (1977) y Rochester y Martin (1979)-, parece expresar con claridad lo que parece ser uno de los déficit más significativos y diferenciadores del lenguaje de los esquizofrénicos: la dificultad para adoptar la perspectiva del otro, durante el proceso de codificación lingüística y, consiguientemente, la dificultad de elaborar discursos eficaces desde un punto de vista comunicativo. 
Junto a la confirmación de déficit globales de carácter semántico y comunicativo en los pacientes esquizofrénicos, los datos del análisis de covarianza demuestran que variables sintácticas tales como la «gramaticalidad de las oraciones» o la "proporción de titubeos en el habla espontánea» no sólo no son irrelevantes para el estudio de la competencia discursiva de este tipo de pacientes sino que pueden, como sugieren Morice e Ingram (1982) y Fraser et al. (1986), ser indicativas de una alteración sobreañadida, no sabemos si específica, en el componente sintáctico del procesamiento lingüístico.

A falta de una confirmación más directa de esta hipótesis, y al objeto de intentar una primera reconstrucción teórica de los procesos afectados en los pacientes diagnosticados de esquizofrenia, consideraremos, sin embargo, que los datos obtenidos, tomados en su conjunto, parecen apoyar, cuando menos, dos tipos de hipótesis:

En primer lugar, la hipótesis (sostenida tanto desde la Lingüística Sistémica -vg. Fawcett, 1972, 1985- como desde la Psicolingǘstica computacional -vg. Steedman y Johnson-Laird, 1980; Zammuner, 1981-) de que el conocimiento que el hablante tiene del oyente y del contexto comunicativo constituye un «nivel de información básico» para la producción de discursos comunicativamente eficaces. Consiguientemente, cualquier alteración que se observe en el uso de índices o marcadores lingüísticos tales como los de foricidad, distancia entre los vínculos de cohesión y sus referentes, etc., puede ser interpretada como reflejo de alteraciones en el uso de este sistema informacional.

En segundo lugar, la hipótesis (sostenida fundamentalmente por Van Dijk, 1980) de que la actividad de producción de los discursos comienza con la elaboración por parte del hablante de una primera representación del «significado provisional e intencionado» del texto (la llamada «macroproposición») que opera como núcleo informativo generador de las unidades representacionales o semánticas más específicas del discurso («subtópicos», "proposiciones individuales», etc.) que sirven a su vez de input directo para el proceso de estructuración superficial de los discursos en sus aspectos sintáctico y morfoléxico (cfr. por ejemplo Davey y Longuet-Higgins, 1976, para una formulación más concreta, en términos computacionales, de esta hipótesis). Desde ese punto de vista, las alteraciones sintácticas en el nivel oracional pueden verse como un subproducto o consecuencia de la mala codificación macroestructural de los discursos, y no, necesariamente, como el resultado de una alteración específicamente morfosintáctica.

Si asumimos, por ejemplo, que la dimensión «calidad representacional de los constituyentes textuales» (tal como ha sido operativizada en nuestro estudio) permite una cierta valoración del grado de desarrollo o elaboración macroproposicional de los discursos, y si interpretamos las otras dimensiones empíricas de nuestro estudio (coherencia, foricidad, cohesión, sintaxis, fluidez) como puntos intermedios de una escala de "profundidad estructural» del discurso y/o de los diferentes niveles o sistemas de procesamiento implicados en la producción de los discursos verbales (fonológico, morfoléxico, sintáctico, semántico y pragmático), los datos obtenidos en nuestro ANOVA parecerían compatibles con la hipótesis de que el hablante esquizofrénico sufre, básicamente, una perturbación en las fases iniciales de planificación del babla que afecta, sobre todo, al "diseño semántico y pragmático global" de su discurso. Subsidiariamente, esta perturbación parece determinar alteraciones de tipo morfosintáctico en la estructura superficial del texto y distorsiones en los aspectos motóricos de la producción del habla (fluidez verbal).

La confirmación de la existencia de diferencias estadísticamente significativas en el análisis de covarianza proporciona, pues, un cierto respaldo em- 
pírico a la hipótesis de que, en los pacientes esquizofrénicos, se da una alteración o trastorno que afecta a los componentes más «centrales» del procesamiento lingüístico (por utilizar la terminología de Fodor, 1986) -es decir, los sistemas menos modulares y autónomos desde un punto de vista funcional- Esta interpretación de los resultados parece compatible con la idea clásica (sostenida tradicionalmente en el contexto clínico) de que son alteraciones «del pensamiento» y no alteraciones específicamente morfosintácticas o léxicas las que explican funcionalmente la incoherencia, desorganización o ininteligibilidad de los discursos esquizofrénicos. Por la misma razón, obligan al investigador a profundizar en la búsqueda de los factores o procesos que, sin ser estrictamente lingüísticos, pueden estar vinculados funcionalmente con estas «fases iniciales de la producción verbal» en las que, al parecer, son más deficitarios los pacientes esquizofrénicos.

$\mathrm{Al}$ análisis de algunos de estos factores y de su posible relación con las alteraciones esquizofrénicas del lenguaje dedicaremos las siguientes secciones.

\section{Parámetros cognitivos y lingüísticos en la explicación de las} alteraciones del discurso de los pacientes esquizofrénicos

Partiendo de la consideración (desarrollada en un trabajo anterior - Belinchón, en este mismo volumen-) de que las alteraciones que se observan en los discursos verbales de los pacientes esquizofrénicos admiten interpretaciones estrictamente lingüísticas (vg. mala utilización de las reglas de composición textual - Chaika, 1982-), pero, también, interpretaciones que las vinculan con déficit más generales en los procesos cognitivos superiores o en el sistema general del procesamiento humano de la información (vg. Rochester, 1978; en este mismo volumen) se analizaron las relaciones de covariación existentes entre las variables lingüisticas diseñadas para el presente trabajo y dos variables cognitivas (la atención sostenida o vigilancia, y la habilidad para solucionar problemas de secuenciación lógica) que, desde diferentes marcos teóricos, pueden considerarse como potencialmente relacionadas con la actividad de planificación y producción de discursos naturales intencionales y complejos.

\section{II.1. Alteraciones esquizofrénicas del discurso y déficit atencional}

Si existe un resultado claro en los datos comentados hasta ahora que justifique una aproximación cognitiva en el estudio del lenguaje esquizofrénico éste es, probablemente, el de que los parámetros lingüísticos que han demostrado tener buena capacidad para la diferenciación estadística de los grupos esquizofrénico y control son parámetros que (teóricamente al menos) están vinculados con los componentes o sistemas más centrales de la producción verbal y, por consiguiente también, con los componentes más interactivos (menos modulares) y menos automáticos del procesamiento lingüístico.

La utilización, en este trabajo, de variables lingüisticas que son indicativas (siquiera de forma aproximativa) de la habilidad de los hablantes en el uso de los niveles de información que controlan la secuencia de sus emisiones lingüisticas ha proporcionado un apoyo relativamente consistente con la hipótesis de que los esquizofrénicos tienen más dificultad que sus controles en el uso de niveles de información cognitivamente complejos tales como las presuposiciones pragmáticas que se refieren al «balance informativo del interlocutor» cuya utilización, durante la producción verbal, tiene un carácter estratégico o controlado. En un análisis ulterior (Belinchón, en preparación) de- 
mostraron que tal dificultad es tanto más evidente cuando el sujeto se enfrenta a tareas (como la narración de historias previamente oídas o la explicación de argumentos relacionados con temas que carecen de referentes directos en el contexto inmediato) que requieren una demanda atencional y/o mnésica relativamente alta.

Vistos desde una perspectiva cognitiva, los resultados comentados parecen admitir una interpretación a partir de la distinción entre «procesamiento automático y controlado» propuesta por Schneider y Shiffrin a finales de los años setenta (Schneider y Shiffrin, 1977; Shiffrin y Schneider, 1977) y permiten, por consiguiente, aventurar una interpretación interferencial ( $y$, por supuesto, provisional) de las alteraciones esquizofrénicas del discurso como alteraciones que se derivan o se relacionan funcionalmente con un déficit de naturaleza atencional.

La hipótesis no es evidente nueva (cfr. Rochester, en este mismo volumen). Sin embargo, es en extremo sugerente. Entre otras cosas, porque da pie a detectar una cierta convergencia entre los resultados obtenidos en nuestros análisis y un conjunto amplio y heterogéneo de datos de otros autores. Por un lado, datos como los de Butterworth (1975, 1980), procedentes del ámbito de la Psicolingüística experimental (análisis cronométrico del habla), que revelan que la determinación de las relaciones conceptuales entre las distintas partes del texto (es decir, la planificación semántica global de las locuciones) tienen una exigencia de recursos atencionales más alta que, por ejemplo, los procesos de codificación sintáctica (a nivel oracional) o la planificación fonoarticulatoria. Por otro, la convergencia respecto a los numerosos resultados experimentales que indican que los esquizofrénicos son deficitarios en tareas que exigen un procesamiento controlado pero no presentan déficit alguno en procesos automáticos o de baja demanda atencional (cfr. Ruiz Vargas, 1985).

Dado que la información obtenida a través del análisis lingüístico no podía sino proporcionar una evidencia harto indirecta sobre la naturaleza de los déficit observados, se optó por reunir evidencia convergente capaz de clarificar, en la medida de lo posible, la viabilidad de esta nueva hipótesis atencional de las alteraciones esquizofrénicas del lenguaje (cfr. Belinchón y Ruiz Vargas, 1985, para un análisis pormenorizado de otras alternativas teóricamente próximas a ésta).

En primer lugar, tratamos de verificar, en nuestra muestra experimental, la validez de la hipótesis que asume, para los esquizofrénicos, una «mala política de asignación de los recursos atencionales» (Kahneman, 1973) y/o «una alteración de la función de control que regula la movilización y distribución de la atención» (Gjerde, 1983). Dicha hipótesis predice una peor ejecución en los esquizofrénicos respecto a sus controles normales en tareas que exigen un procesamiento controlado o que son difícilmente automatizables.

En segundo lugar, tratamos de verificar si se daban o no relaciones de covariación entre los índices de la ejecución atencional y los índices lingüísticos utilizados en la valoración de la coherencia de los discursos. De existir tal covariación, suponíamos, la interpretación de las alteraciones del discurso como alteraciones subsidiarias a déficit más generales en el procesamiento controlado de la información podría obtener un respaldo empírico relativamente consistente. De lo contrario, cabría rechazar tal interpretación y considerar que ambos tipos de alteración obedecen a sistemas de procesamiento funcionalmente diferentes y no necesariamente relacionadas.

La medida utilizada para la verificación del déficit atencional fue (como ya se expuso en la sección de "Método») una medida directa del funcionamiento controlado: una tarea de vigilancia (el C.P.T.) que, por sus características, hace imposible la automatización de la respuesta (cfr. Ruiz Vargas y Bo- 
tella, 1987). Las variables lingüísticas analizadas fueron las mismas que demostraron su buen poder discriminativo en el análisis de covarianza comentado en la sección anterior.

\section{II.2. Resultados y discusión de los datos atencionales}

La aplicación de un ANOVA de un solo factor para grupos independientes (mediante un programa BMDP7D) a los datos procedentes del CPT, confirmaron de forma rotunda la primera de nuestras predicciones, a saber: la peor ejecución de los esquizof rénicos en la tarea de vigilancia $(F(1,38)=22,45$; $\mathrm{p}<.001)$.

El cálculo de los «coeficientes de correlación producto-momento de Pearson» entre las puntuaciones de los sujetos en la variable atencional y en las lingüísticas (ver tabla XIX) arrojó, por su parte, resultados consistentes con la hipótesis de que la capacidad de los sujetos para mantener focalizada la atención en la tarea guarda una estrecha relación con el grado de organización o desorganización estructural de los discursos.

A primera vista, los datos no permiten identificar con claridad los niveles de demanda atencional requeridos en cada nivel de la codificación lingüística y, por esta razón, no cabe formular a partir de estos resultados hipótesis muy específicas relativas al grado de control que requieren los procesos supuestamente representados por los índices lingüísticos de nuestro estudio. Desde un punto de vista teórico, sin embargo, los datos permiten no descartar por el momento la hipótesis de que las alteraciones esquizofrénicas del discurso puedan estar más relacionadas con una alteración del sistema global del procesamiento de información que con una alteración específica del procesamiento lingüístico. Metodológicamente, además, los resultados, aunque estadísticamente son modestos, tienen un alcance que creemos es poco desdeñable. Entre otras cosas, porque las variables lingüísticas que proceden de análisis ex-

\section{TABLA XIX}

Correlaciones significativas (probabilidad de error menor al 5 por 100) entre las variables lingüísticas $y$ las puntuaciones del CPT

\begin{tabular}{lrl}
\hline & $\mathrm{CPT}$ \\
\hline \multicolumn{1}{c}{ palabras por oración. } & $.36 \quad(\mathrm{p}<.05)$ \\
$\quad$ frases por oraciones. & $.43 \quad(\mathrm{p}<01)$ \\
Porcentaje de frases bien construidas. & $.56 \quad(\mathrm{p}<001)$ \\
Porcentaje de oraciones simples. & $-.33 \quad(\mathrm{p}<05)$ \\
$\quad$ vínculos cohesión. & $.48 \quad(\mathrm{p}<01)$ \\
Porcentaje de referencia. & $.37 \quad(\mathrm{p}<05)$ \\
$\quad$ distancia cohesión. & $.43 \quad(\mathrm{p}<01)$ \\
$\quad$ GNF. & $.37 \quad(\mathrm{p}<05)$ \\
Pordentaje de exófora. & $-.41 \quad(\mathrm{p}<02)$ \\
Porcentje referente no claro. & $-.37 \quad(\mathrm{p}<05)$ \\
Porcentaje de propos. de vínculos explícitos. & $.48 \quad(\mathrm{p}<01)$ \\
Porcentaje de propos. de neceisiones explícitos. & -.35 & $(\mathrm{p}<05)$ \\
Porcentajes no coherentes. & -.40 & $(\mathrm{p}<02)$ \\
Porcentajes de extradicscursivas & -.34 & $(\mathrm{p}<05)$ \\
NE + NC + EXTR. & $-.47 \quad(\mathrm{p}<01)$ \\
Módulos I & $.38 \quad(\mathrm{p}<05)$ \\
Módulos III & $-.43 \quad(\mathrm{p}<01)$ \\
Módulos VI & $-.60 \quad(\mathrm{p}<001)$ \\
AE ${ }_{1}$. & $.39 \quad(\mathrm{p}<02)$ \\
Congruencia de la narración con el material original. & $.56 \quad(\mathrm{p}<.001)$ \\
\hline
\end{tabular}


perimentales de los discursos como el nuestro nunca antes habían sido analizados conjuntamente con otras variables cognitivas (los estudios de Mintz y Alpert (1972) y Heilbrun (1980) sobre la relación entre variables cognitivas y perceptuales con la presencia de alucinaciones auditivas, o el informe de Manschreck et al. (1981) sobre las relaciones de la conducta motriz y el desorden formal del pensamiento, se han desarrollado en contextos teóricos muy diferentes y, por tanto, no pueden considerarse, en rigor, como precedentes reales del trabajo aquí realizado).

\section{II.3. Alteraciones esquizofrénicas del discurso y alteraciones en la babilidad para solucionar problemas de ordenación lógica}

Los datos presentados anteriormente sugieren la posibilidad de vincular funcionalmente los déficit lingüísticos de los esquizofrénicos (detectados a través del análisis de sus producciones verbales) con déficit específicos, de carácter atencional, que teóricamente estarían en la base de las alteraciones en el procesamiento controlado de la información de este tipo de pacientes.

Resulta evidente que, desde el enfoque dominante en la actual Psicología Cognitiva (el enfoque del procesamiento de la información -cfr. Lachman et al., 1979; Delclaux y Seoane, 1982-), este tipo de interpretaciones no sólo es plausible sino que es también deseable. Sin embargo, resulta evidente que, en el momento actual, la Psicología del Lenguaje continúa teniendo un carácter interdisciplinar y multiparadigmático (cfr. Mayor y Gil, 1984) que, por el momento, hace también posible (e incluso necesaria) la búsqueda de explicaciones de la actividad linguística humana fuera de este enfoque teórico concreto.

Asumiendo, por tanto, esta necesidad, y utilizando como punto de referencia el intento de De Beaugrande (1980) por vincular teóricamente la actividad lingüística de producción de discursos con variables cognitivas no derivadas estrictamente del enfoque del P.I. (ver tabla XX), se analizaron también las relaciones de covariación existentes entre los índices lingüísticos diseñados para la comparación de los sujetos esquizofrénicos y controles y una variable - la capacidad para solucionar problemas- cuyo interés para la investigación de los discursos ha comenzado a ser destacada en algunas investigaciones recientes (vg. Hinds, 1979; Levy, 1979; Hobbs y Evans, 1980).

\section{TABLA XX}

Competencias generales cognitivas relacionadas con la producción de los discursos (adaptado de De Beaugrande, 1980a; págs. 125-126)

1) Habilidad para la solución de problemas.

2) Habilidad para la planificación de acciones.

3) Habilidad para inferir el nivel de habilidad de otras personas (los interlocutores) en la solución de problemas y la planificación de acciones.

4) Habilidad para generar, probar y revisar hipótesis sobre los inputs actuales y su relevancia específica respecto a contextos más amplios.

5) Facilidad de procesamiento para los inputs o outputs esperados o probables.

6) Profundidad de procesamiento para los inputs o outputs no esperados o improblables.

7) Habilidad para reducir la complejidad de los inputs (p. ej., mediante procesos de esquematización).

8) Capacidad para localizar selectivamente la atención.

9) Capacidad para mantener una continuidad en la experiencia. 
En nuestro caso, la medida de la capacidad de los sujetos para la solución de problemas se obtuvo a partir de la administración de la subescala «Historietas» de la Escala manipulativa del WAIS. Como se sabe, esta tarea requiere de los sujetos que ordenen unidades de significado (viñetas) en una secuencia lógico-temporal. La tarea no permite medir, ciertamente, algunos aspectos de la habilidad para solucionar problemas (como la capacidad de definir submetas, o la de seleccionar entre estrategias alternativas) que parecen también interesantes, como analogía, para una caracterización teórica de la producción de discursos verbales (producción de discurso = solución de una serie de subproblemas de organización semántica y morfosintáctica que deben desembocar en una estructura lingüística tal que «realice» el «acto de habla global» del hablante y que pueda ser eficazmente comprendida por el oyente). Sin embargo, como primera aproximación al problema, parecía suficientemente útil.

Los datos obtenidos tanto en la comparación de los dos grupos de sujetos (ANOVA de un factor con medidas independientes) como en el análisis de las correlaciones con las variables lingüísticas (análisis de las correlaciones producto-momento de Pearson), arrojaron resultados bastante similares a los obtenidos en el análisis de las puntuaciones obtenidas en la tarea del C.P.T. (ver tabla XXI). Los esquizofrénicos, por una parte, demostraron su mayor dificultad para la solución correcta de los problemas del WAIS $(F(1,38)=13,49 ; \mathrm{p}<.001)$. A su vez, la dimensión se mostró como significativamente relacionada, entre otras, con variables como la «proporción de oraciones yuxtapuestas e incrustadas» (es decir, vinculadas gramaticalmente sin nexo sintáctico superficial) $(\mathrm{r}=-.37 ; \mathrm{p}<.05)$, el «promedio de vínculos de cohesión» $(\mathrm{r}=.45 ; \mathrm{p}<.01)$ o la «proporción de proposiciones con vínculo lógico explícito» $(\mathrm{r}=.42 ; \mathrm{p}<.01)$ que son claramente indicativas de la capacidad de los sujetos para establecer relaciones estructurales apropiadas (o, si se prefiere, gramaticalmente aceptables) entre las unidades lingüísticas de sus discursos.

\section{TABLA XXI}

Correlaciones significativas (probabilidad de error, inferior al 5 por ciento) obtenidas en la comparación de las puntuaciones en la tarea de "Historietas" del WAIS y las variables lingüísticas

\begin{tabular}{|c|c|c|c|}
\hline & & \multicolumn{2}{|c|}{ Historias } \\
\hline 277) & palabras por oración. & .48 & $\mathrm{p}<.01$ \\
\hline 278) & frases por oración. & .47 & $\mathrm{p}<.01$ \\
\hline 284) & Porcentaje de proposiciones sin nexo. & -.37 & $\mathrm{p}<.05$ \\
\hline 288) & vínculos de cohesión. & .45 & $\mathrm{p}<.01$ \\
\hline 294) & GNF. & .34 & $\mathrm{p}<.05$ \\
\hline 301) & Porcentaje de proposiciones de vínculos explícitos & .42 & $\mathrm{p}<.01$ \\
\hline 303) & Porcentaje de proposiciones de necesarias explícitos & -.47 & $\mathrm{p}<.01$ \\
\hline 304) & de proposiciones no coherentes. & & \\
\hline 307$)$ & $\mathrm{NE}+\mathrm{NC}+\mathrm{EXTR}$ & -.34 & $\mathrm{p}<.05$ \\
\hline 308) & Módulos tipo I. & .50 & $\mathrm{p}<.01$ \\
\hline 310) & Módulos tipo III. & -.40 & $\mathrm{p}<.02$ \\
\hline 311) & Porcentaje de Módulos tipo VI. & & \\
\hline 318) & Comprensión de narraciones con material original. & .48 & $\mathrm{p}<.01$ \\
\hline
\end{tabular}

\section{Alteraciones del lenguaje y variables clínicas}


real de la perturbación esquizofrénica en lo que concierne a la producción de los discursos, pero, de forma consistente, ponen de manifiesto la necesidad de interpretar tal perturbación como un fenómeno complejo en el que pueden intervenir variables de índole muy diversa. Entre ellas, y mientras no se demuestre empíricamente lo contrario, no pueden descartarse las variables que son indicativas del nivel de perturbación general que sufren los pacientes, es decir: las variables clínicas.

La hipótesis (sostenida, entre otros, por Rochester y Martin, 1979) de que, por debajo de unos niveles mínimos de «severidad clínica en la sintomatología», el lenguaje de los pacientes esquizofrénicos puede no ser diferenciable del de los sujetos normales, continuaba resultando plausible a pesar de que tanto el análisis discriminante como el de varianza con covariados habían revelado diferencias significativas entre nuestros dos grupos experimentales. Por esta razón, optamos por realizar un análisis empírico complementario relacionando las puntuaciones obtenidas en el análisis lingüístico con las obtenidas en variables tales como el «nivel global de la sintomatología», el «nivel de cronicidad de la enfermedad» o el «tiempo de hospitalización» que, presumiblemente, podían estar influyendo en el nivel de desorganización de los discursos (cfr. Harrow y Quinlan, 1985).

Dado el carácter exploratorio del estudio, la metodología utilizada fue también de carácter correlacional («coeficiente producto-momento de Pearson», calculado mediante el programa BMDP7D).

Tal y como se observa en la tabla XXII, la relación de covariación de las variables «años de tratamiento», «distancia en años desde el primer ingreso» y «días de hospitalización en el momento de recogida de las muestras lingüísticas» con las variables lingüísticas del estudio fue, salvo raras excepciones, escasamente significativa. Algunas de las correlaciones obtenidas, además, contradicen abiertamente observaciones como las de Harrow y Quinlan (1985) en el sentido de que se da una disminución drástica de las alteraciones lin-

TABLA XXII

Correlaciones significativas entre las variables lingüisticas $y$ clinicas del estudio

\begin{tabular}{|c|c|c|c|c|}
\hline & $\begin{array}{c}\text { Años de } \\
\text { tratamiento } \\
(\mathrm{N}=20)\end{array}$ & $\begin{array}{c}\text { Años desde } \\
1.0 \text { ingreso } \\
(\mathrm{N}=20)\end{array}$ & $\begin{array}{l}\text { Días de } \\
\text { estancia } \\
(N=20)\end{array}$ & $\begin{array}{c}\text { BPRS } \\
(\mathbf{N}=33)\end{array}$ \\
\hline $\begin{array}{l}\text { palabras. } \\
\text { frases. } \\
\text { oraciones. } \\
\text { errores fluidez. } \\
\text { frases bien construidas. } \\
\text { oraciones simples. } \\
\text { Porcentaje de subordinadas. } \\
\text { Porcentaje de endóforas. } \\
\text { Porcentaje de exóforas. } \\
\text { Porcentaje de exófo- } \\
\text { ras+implícitas. } \\
\text { Porcentaje de proposición } \\
\text { no coherente } \\
\text { NE+NC+EXTRAD. } \\
\text { Porcenłaje de módulos } \\
\text { tipo II. } \\
\text { Porcentaje de módulos } \\
\text { tipo VI. } \\
\text { Asociaciones explícitas } \\
\text { Porcentaje de módulos re- } \\
\text { cordados. } \\
\text { Desarrollo argumental. }\end{array}$ & $\begin{array}{l}.63(\mathrm{p}<.01) \\
.55(\mathrm{p}<.02) \\
.58(\mathrm{p}<.02)\end{array}$ & $\begin{array}{l}.53(\mathrm{p}<.05) \\
.54(\mathrm{p}<.05)\end{array}$ & $\begin{array}{r}-.53(\mathrm{p}<.05) \\
.52(\mathrm{p}<.05)\end{array}$ & $\begin{array}{r}-.42(\mathrm{p}<.05) \\
.39(\mathrm{p}<.05) \\
.47(\mathrm{p}<.02) \\
.47(\mathrm{p}<.02) \\
.37(\mathrm{p}<.05)\end{array}$ \\
\hline
\end{tabular}


güísticas de los pacientes entre la primera y la segunda semana de su ingreso en la unidad de asistencia psiquiátrica.

Las correlaciones con el «nivel global de severidad de la sintomatología» son, sin embargo, algo más consistentes. A partir de los resultados recogidos en la tabla XXII, es posible afirmar (con cautela, una vez más) que existe una relación de covariación de signo negativo en el nivel general de la sintomatología y parámetros lingüísticos como la complejidad estructural o la organización sintáctica de las oraciones, que pueden ser detectados en los discursos desviados de los esquizofrénicos. Paralelamente, se observa una relación de signo positivo respecto a la variable «nivel de referencias exofóricas» (que indirectamente resta cohesión al propio discurso) y respecto a los indicadores lingüísticos de incoherencia proposicional, incongruencia temática con los referentes de la tarea e incongruencia macroestructural.

Tales relaciones de covariación indican, si no las interpretamos mal, que el nivel de desorganización e incoherencia del lenguaje es, tal y como tradicionalmente han interpretado algunos clínicos (vg. Harrow y Quinlan, 1985; Andreasen et al., 1985), subsidiaria, al menos en parte, del nivel de exacerbación de la sintomatología psicótica. No obstante, en la medida en que los pacientes que componían nuestra muestra no obtuvieron puntuaciones muy altas en el B.P.R.S. ( $\overline{\mathrm{x}}=38,05$, con un rango 19-67 puntos, sobre un máximo de 126 puntos de la escala) cabe sospechar que el nivel de agudización de la sintomatología global no permite explicar en su totalidad la perturbación lingüística (en cualquier caso, tampoco aportaría dato alguno respecto a los mecanismos psicolingüísticos afectados por tal perturbación).

En tanto no se realice, por ejemplo, un análisis de regresión múltiple (a partir de las puntuaciones de un grupo más amplio de sujetos) o un estudio de seguimiento que informe sobre la evolución del trastorno lingüístico en los pacientes (Guimón, Ruiz Vargas, Belinchón, Grijalbo, en preparación), no será posible, obviamente, determinar cuál es el "peso" explicativo real de esta variable en relación con el grado de desorganización estructural y/o de ineficacia referencial de los discursos esquizofrénicos. Por el momento, simplemente, cabe afirmar que el «nivel global de severidad de la sintomatología» resulta ser una variable de interés tanto metodológico (selección/control de los grupos experimentales) como teórico, para el estudio de problemas tales como el carácter episódico o, por el contrario, permanente, de la perturbación lingüística de este tipo de pacientes.

\section{DISCUSION GENERAL Y CONCLUSIONES}

Los resultados presentados en las secciones anteriores parecen proporcionar un claro apoyo empírico a dos hipótesis que bien podrían considerarse como nucleares en el ámbito de la investigación psicolingüística de las alteraciones esquizof rénicas del lenguaje. En primer lugar, la hipótesis de que los discursos de estos pacientes pueden ser diferenciados de los de los sujetos normales a partir de un análisis lingüístico de carácter estructural. En segundo lugar, la hipótesis de que estas diferencias lingüísticas pueden ser vinculadas teóricamente con déficit y alteraciones cognitivas que, presumiblemente, están relacionadas con la producción verbal de los discursos.

En nuestro estudio, los textos producidos por los sujetos experimentales fueron analizados en dimensiones que constiruían desarrollos empíricos o aplicaciones de la noción general de coberencia lingǘstica. Los índices utilizados valoraban aspectos micro y macroestructurales de los discursos y eran indica- 
tivos de rasgos, dimensiones o habilidades de tipo sintáctico, semántico y pragmático.

Globalmente considerados, los resultados obtenidos en los análisis han demostrado una fuerte consistencia interna respecto al valor diagnóstico de los indicadores lingüísticos y a su utilidad para la formulación de hipótesis relativas a la naturaleza de la alteración esquizofrénica. En primer lugar, los resultados demostraron la superioridad discriminativa de los índices que valoraban el grado de conexión explícita o admisible de las unidades sintácticas o semánticas del discurso (gramaticalidad de las oraciones, cohesión referencial, léxica y sintáctica de las unidades superficiales del texto, coherencia en la vinculación entre proposiciones en el nivel de la estructura profunda, organización macroestructural de los constituyentes textuales, etc.). En segundo lugar, los resultados demostraron claras diferencias entre el grupo de esquizofrénicos y el control en el uso de las claves lingüísticas que facilitan al interlocutor la recuperación o identificación de los referentes del discurso (tanto micro como macroestructurales): la utilización de grupos nominales con referentes no ambiguos, la utilización de vínculos de cohesión con referentes textualmente próximos, o la construcción de constituyentes textuales capaces de comunicar la información temática más relevante y de facilitar al oyente la elaboración de un «esquema semántico» sobre el que apoyar su proceso de interpretación de los discursos.

La confirmación (a través de análisis de diferencias y análisis discriminantes) de que los esquizofrénicos pueden sufrir una alteración en los componentes «centrales» de la producción verbal (los responsables del diseño semántico o pragmático - macroestructural- de los discursos), y la confirmación posterior de la existencia de relaciones de covariación entre los indicadores linguísticos de esta perturbación y las puntuaciones obtenidas en tareas que miden la habilidad de los sujetos para realizar procesos de carácter estratégico o controlado, y la habilidad para solucionar problemas de ordenación lógico-temporal, han dado pie fácil a un conjunto sugerente de hipótesis relativas tanto al perfil de «competencias» o «incompetencias» del hablante esquizofrénico como al proceso de "producción verbal de discursos naturales» como tal. Además, los datos presentados permiten entrever la necesidad de desarrollar líneas de investigación específicas que profundicen en los aspectos metodológicos de este tipo de investigaciones (vg. el tipo de tarea utilizado en la elicitación de las muestras lingüísticas y el tipo de índices utilizados en la comparación de las mismas). Sin embargo, parece necesario extremar las precauciones a la hora de valorar la importancia de los resultados obtenidos $y$, sobre todo, no intentar extraer conclusiones definitivas sobre la naturaleza de los déficit lingüísticos de este tipo de pacientes.

Primero, porque la comparación entre grupos realizada en este estudio no incluyó un grupo control de pacientes psicóticos capaz de diferenciar empíricamente el componente propiamente «esquizofrénico» y el componente «psicótico» de las producciones de nuestros pacientes. Segundo, porque los índices y tareas utilizadas en este estudio no son sino primeros intentos de operativización de nociones teóricas complejas (como la de «coherencia») que parecen requerir una reflexión teórica y una elaboración metodológica claramente más sofisticada que las que han servido de base a la realización de este trabajo. Tercero, porque los datos que proceden de un análisis estructural de los discursos, aunque indudablemente pueden proporcionar una información muy valiosa, no indican más que de forma indirecta cuáles son los procesos implicados en la producción de los discursos y cuáles las posibles causas de su mal funcionamiento (cfr. por ejemplo, De Beaugrande, 1979).

El proceso de realización de este trabajo ha ido evidenciando alguna de 
las enormes restricciones que el método científico impone a las descripciones del lenguaje natural cuando éstas tratan de ser «ecológicamente válidas» y, a la par, «teóricamente útiles». A la vez, dicho trabajo ha ido evidenciando algunas de las limitaciones heurísticas que parece tener este tipo de descripciones. Aun así, los resultados obtenidos permiten suponer que este modo de enfocar el estudio empírico de los procesos de producción verbal puede ser viable y útil para la investigación de las perturbaciones del lenguaje, en general, y para la del «lenguaje esquizofrénico» en particular. Queda ahora a expensas de nuevas investigaciones, la confirmación empírica o no de estas primeras conclusiones.

\section{Nota:}

Agradezco muy sinceramente a Pilar Carrera, Nuria Carriedo, Carmen Ballesteros y Monserrat Sanz su colaboración en la transcripción y codificación lingüística de los protocolos utilizados en este estudio.

\section{Resumen}

Partiendo de algunos de los conceptos y métodos desarrollados por la Psicolingüistica experimental de orientación textual, se analizaron empíricamente 160 textos producidos por sujetos esquizofrénicos y normales para determinar si bay o no en los esquizofrénicos déficit específicos en la producción verbal y para comprobar, en su caso, cuáles son los niveles de procesamiento más afectados por tal déficit y cuáles son posibles determinantes funcionales. Los resultados del análisis de siete dimensiones lingü̈sticas, derivadas de la noción de "coberencia textual", confirmaron la existencia en los esquizofrénicos de alteraciones relacionadas con el diseño semántico y pragmático de los discursos, que guardan una estrecha relación con la mala política de asignación de recursos atencionales en la tarea, la dificultad para resolver problemas de ordenación lógica y el nivel global de severidad de la sintomatología de los pacientes. Los resultados confirmaron, asimismo, la utilidad de este tipo de estudios para la interpretación teórica de las per. turbaciones del lenguaje y la necesidad de profundizar en aspectos metodológicos tales como los tipos de tareas, los grupos experimentales y los indices de medida utilizados en el análisis de las muestras lingüisticas.

\section{Abstract}

One bundred sixty texts produced by schizophrenic and normal subjects were analyzed in order to find specific verbal production deficits in schizophrenics, and to specify the most disturbed levels of processing and their possible functional determinants. Analyses based on theoretical models of textual Psycholinguistics were carried out. The analysis of seven linguistic dimensions - derived from the notion of "textual coherence" - made clear the existence of disturbances related to semantic and pragmatic planning of discourses in schizophrenic subjects. Such disturbances are explained in relation to an inadequate allocation policy of attentional resources, a difficulty for solving problems of logical ordering, and the overall level of simptomatology. Results also made evident the utility of this type of approach for the theoretical interpretation of language disturbances, as well as the need for further methodological issues such as type of tasks, selection of experimental groups and the measures for linguistic analysis.

\section{Referencias}

ALLEN, H. A.: «Do positive symptom and negative symptom subtypes of schizophrenia show qualitative differences in language production?». Psychological Medicine, 1983, 13, 787-797.

ANDREASEN, N. C.: «Thought, language and communication disorders. 1 . Clinical assessment, definition of terms, and evaluation of their reliability». Arch. of Gen. Psychiat., 1979a, 36, 1315-1323.

ANDRENSEN, N. C.: «Thought, language and communication disorders. 2. Diagnostic significance». Arch. of Gen. Psychiat., 1979b, 36, 1325-1330.

ANDREASEN, N. C., y Grove, W.: «The relationship between schizophrenic language, manic language and aphasiax. En J. Gruzelier y P. Flor-Henry (Eds.): Hemisphere asymmetries and function in psychopatbology. Elsevier: North-Holland Press, 1979, págs. 373-390.

ANDREASEN, N. C.; HofFMAN, R. E., y GROVE, W. M.: «Mapping abnormalities in language and cognition». En M. Alpert (Ed.): Controversies in schizophrenia: Changes and constancies. Nueva York: The Guilford Press, 1985, págs. 199-227. 
AYLWARD, E.; WALKER, E., y BETTES, B.: eIntelligence in schizophrenia: Meta-analysis of the research». Schizophrenia Bulletin, 1984, 10 (3), págs. 430-459.

BAGGETT, P.: «tructurally equivalent stories in movie and text and the effect of the medium on recall». J. of Verb. Learn. and Verb. Behav., 1979, 18, 319-332.

Baron-Cohen, S.; Leslie, A. M. y Frith, U.: «Does the autistic child have a "theory of mind"? Cognition, $1985,21,37-46$

BASTIDE, R.: Sociología de las enfermedades mentales. (3." ed.). México: Siglo XXI 1976.

BeAugrande, R. DE: «Moving from product toward process». College Composition and Communication, 1979, págs. 357-363.

BeAugrande, R. DE: Text, discourse and process. Toward a multidisciplinary science of texts. New Jersey: Ablex Publ. Co., 1980.

BeunCHON, M.: «Lenguaje». En J. M. Ruiz-Vargas (Ed.): Esquizofrenia: Un enfoque cognitivo, cap. 7. Madrid: Alianza Psicología, 1987.

BelinChÓN, M.: «Producción verbal». En I. Delclaux, A. Rivière y M. Carretero (Eds.). Procesos cognitivos, vol. 2. Madrid: Morata (en prensa).

BELINCHÓN, M.: «Cuestiones metodológicas de la investigación sobre la producción de los discursos: La variable "tarea"» (en preparación).

Belinchón, M., y Ruiz-VARGas, J. M.: «El papel de las hipótesis atencionales en la explicación del lenguaje esquizofrénico: Una revisión». Rev. Psicol. Gral. y Apli., 1985, 40 (4), 757-776.

Bleuler, E.: Demencia Precoz o el grupo de las esquizofrenias. Buenos Aires: Hormé, 1960.

BLEULER, M.: «Inconstancy of schizophrenic language and symptoms». The Behav. and Brain Sci., 1982, S, pág. 591.

BUTTERWORTH, B.: «Hesitation and semantic planning in speech». J. of Psycholinguistic Research, 1975, 4 (1), $75-87$

BUTTERwORTH, B.: «Evidence from pauses in speech». En B. Butterworth (Ed.): Language production, Vol. 1. Londres: Academic Press, págs. 155-176.

Carroll, D. W.: Psychology of language. Monterey: Brooks/Cole Publ. Co., 1986.

CLARK, H., y ClARK, E. V.: Psychology and language: An introduction to psycholinguistics. Nueva York: Harcourt Brace Jovanovich, 1978.

COHEN, B. D.: «Referent communication in schizophrenia». En S. Schwartz (Ed.): Language and cognition in schizopbrenia. Hillsdale, N. J.: LEA, 1978, págs. 1-33.

COHEN, B. D., y CAMHI, J.: «Schizophrenic performance in a word communication task». J. of Abnormal Psycho$\log y, 1967,72,240-246$

COHEN, B. D.; NACHMANI, G., y RosenBERG, S.: «Referent communication disturbances in acute schizophrenic». J. of Abnormal Psychology, 1974, 83, 1-13.

CHAFE, W. L.: «The deployment of consciousness in the production of a narrative». En W. L. Chafe (Ed.): The pear stories: Cognitive, cultural and linguistic aspects of narrative pruduction. Hillsdale, N. J.: LEA, 1980, págs. 9-50.

ChAIKA, E.: «A linguistic looks at "schizophrenic" language». Brain E Language, 1974, 1, 257-276.

CHAIKA, E.: «Schizophranic speech, slips of the tongue, and jargonaphasia: A reply to Fromkin and to Lecours and Vanier-Clement». Brain \& Language, 1977, 4, 464-475.

CHAIKA, E.: «A unified explanation for the diverse structural deviations reported for adult schizophrenics with disrupted speech». J. of Communication Disorders, 1982, 15, 167-189.

Chalka, E., y AleXander, P.: «The Ice Cream Stories: A study in normal and psychotic narration». Comunicación presentada en «The Linguistic Society of America's Annual Meeting» en San Diego, 1982.

ChaIKA, E., y LAMBE, R.: «The locus of dysfunction in schizophrenic speech». Schizophrenia Bulletin, 1985, 11 (1), 8-15

Chapman, L. J.; Chapman, J. P., y Miller, G. A.: «A theory of verbal behavior in schizophrenia». En B. Maher (Ed.): Progress in experimental personality research, vol. 1. Nueva York: Academic Press, 1964.

DAveY, A. C., y Longuet.Higgins, H. C.: «A computational model of discourse production». En R. N. Campbell y Ph. T. Smith (Eds.): Recent advances in the psychology of language. Nueva York: Plenum Press, 1976, págs. 125-136.

Delclaux, I., y Seonne, J.: Psicología cognitiva y procesamiento de la información. Madrid: Pirámide, 1982.

FAWCETT, R. P.: «Systemic functional grammar in a cognitive model of language». Comunicación presentada en el «Meeting of the Linguistic Association of Great Britain» (manuscrito no publicado); Sheffield, 1972.

FAwCETT, R. P.: Systemic Linguistics: Theory and applications. Batsford Publications, 1985.

FiNE, J., y BARTOLUCCI, G.: «Cohesion and retrieval categories in normal and disturbed communication: A methodological note». Discourse Processes, 1981, 4, 267-270.

FLEKKOY, J.: *Association changes in hospitalized schizophrenics during a 16 year period». Neuropsychobiology, $1975,1,47-58$.

FODOR, J.: La modularidad de la mente. Madrid: Morata, 1986.

Foss, D. J., y HAKEs, D. T.: Psycholinguistics: An introduction to the Psychology of language. New Jersey: Prentice-Hall, Inc., 1978.

Fraser, W. I.; KING, K. M.; Thomas, Ph., y Kendell, R.: «The diagnosis of schizophrenia by language analysis». Br. J. of Psychiatry, 1986, 148, 275-278.

Garcia-Albea, J. E.: Prólogo a la edición española del libro de J. A. Fodor: La modularidad de la mente. Madrid: Morata, 1986.

Garcia-Albea, J. E.; IGon, J. M., y SANCHEZ-Bernardos, M. L.: «Nuevas perspectivas en el estudio de la comprensión y la producción del lenguajen. En M. Yela (Ed.): Estudios sobre inteligencia y lenguaje. Madrid: Pirámide, 1987.

GJeRde, W. M., y AndReasen, N. C.: «Attentional capacity dysfuction and arousain schizophrenic». Psychological Bulletin, 1983, 93, 57-72.

Grove, W. M., y ANDREASEN, N. C.: «Language and thinking in psychosis. Is there an input abnormality?» Arch. of Gen. Psychiat., 1985, 42 (1), 26-32. 
Guimón, J.; Ruiz-VARGas, J. M.; Belinchón, M., y GRIjalbo, J.: «Evolución de los déficit cognitivos y lingüísticos en las esquizofrenias». Informe de investigación financiado por la CAICYT (en preparación).

HaRrow, M., y QuINLAN, D. M.: Disorders thinking and schizophrenic psychopatbology. Nueva York: Gardner Press, Inc., 1985.

Heilbrun, A. B.: «Impaired recognition of self-expressed thought in patients with auditory hallucinations». $J$. of Abnormal Psychology, 1980, 89, 728-736.

HINDS, J.: «Organizational patterns in discourse». En T. Givon (Ed.): Syntax and Semanticr. Vol. 12. Nueva York: Academic Press, 1979, págs. 135-157.

HobBS, J. R., y EvaNS, D. E.: «Conversation as planned behavior». Cognitive Science, 1980, 4, 349-377.

HoffMan, R. E.; KIRSTEIN, L.; STOPEK, S., y CiCHETTI, D. V.: «Apprehending schizophrenic discourse: A structural analysis of the listener's task». Brain E Language, 1982, 15, 207-233.

HoFFMAN, R, y SLEDGE, W.: «A microgenetic model of paragrammatisms produced by a schizophrenic speaker». Brain E Language, 1984, 21, 147-173.

HoffMAN, R. E.; STOPEK, S., y ANDREASEN, N. C.: «A comparative study of manic versus schizophrenic speech disorganization». Arch. of Gen. Psychiat. (en prensa).

HYMOwITZ, P., y SPOHN, H.: "The effects of anti-psychotic medication on the linguistic ability of schizophrenics». J. of Nervous and Mental Disease, 1980, 168, 287-296

Kahneman, D.: Attention and effort. New Jersey: Prentice-Hall, Englewood Cliffs, 1973.

KINTSCH, W., y VAN DijK, T. A.: «Toward a model of text comprehension and production». Psychological Review, 1978, 85 (5), págs. 363-394.

KLECKA, W. R.: Discriminant analysis. Londres: Sage Publications, 1980.

Kraepelin, E.: Dementia praecox and paraphrenia. Robert E. Krieger Publish. Co. Inc. Huntington, Nueva York (sobre la edición facsímil de 1919), 1971.

LACHMAN, R.; LACHMAN, J. L., y ButTERFIELD, E. C.: Cognitive psychology and information processing: An introduction. Hillsdale, N. J.: LEA, 1979.

LEvy, D. M.: «Communicative goal and strategies: Between discourse and syntax», en T. Givon (ed.): Syntax and Semantics. Vol. 12. Nueva York: Academic Press, 1979, págs. 183-210.

ManCuSO, J. C.; SARBIN, T. R., y HeERdT, W. A.: «Schizophrenic language: An ephemeron hiding an ephemeron». The Behav. E Brain Sci., 1982, 5, 605-607.

Manschreck, T. C.; Maher, B. A.; Rucklos, M., y White, M. T.: «The predictability of thought disordered speech in schizophrenic patients». Brit. J. Psychiat., 1979, 134, 595-601.

MANSCHRECK, T. C.; MAHER, B. A., y RUCKLOS, M. E.: «Cloze procedure and written language in schizophrenia». Language and Speech, 1980, 23, 323-328.

ManschreCK, T. C.; MAHER, B. A., y RUCKLos, M. E.: «Formal thought disorder, the type-token ratio and voluntary motor movement in schizophrenia». Br. J. of Psychiat., 1981, 139, 7-15.

MANSChRECK, T. C.; MAHER, B. A.; HoOver, T. M., y AMES, D.: «The type-token ratio in schizophrenic disorders: Clinical and research value». Psychological Medicine, 1984, 14, 151-157.

Mayor, J.: «Texto y discurso». En J. Mayor (Ed.): Psicología del Pensamiento y el Lenguaje. Vol. 1. Madrid: Serv. Public. UNED, 1984, págs. 251-289.

MAYOR, J., y GiL, G.: «Psicología del lenguaje». En J. Mayor (Ed.): Psicología del Pensamiento y el Lenguaje. Vol. 1. Madrid: Serv. Public. UNED, 1984, págs. 7-35.

MINTZ, S., y ALPERT, M.: «Imagery vividness, reality testing, and schizophrenic hallucination», J. of Abnormal Psychology, 1972, 79, 310-316.

MORICE, R., e INGRAM, J. C. L.: «Language analysis in schizophrenia: diagnostic implications». Australian and New Zealand Journal of Psychiatry, 1982, 16, 11-21.

P^vY, D.: «Verbal behavior in schizophrenia: A review of recent studies». Psychological Bulletin, 1968, 70, 164-178.

Reich, S. S., y CutTing, J.: «Picture perception and abstract thought in schizophrenia». Psychological Medicine, $1982,12,91-96$.

ROCHESTER, S. R.: "Are language disorders in acute schizophrenia actually information processing problems?». J. Psychiat. Research, 1978, 14, 275-283.

RoCHESTER, S. R., y MARTIN, J. R.: Crazy talk: A study of the discourse of schizophrenic speakers. Nueva York: Plenum Press, 1979.

ROCHESTER, S. R.; MARTIN, J. R., y Thurston, S.: «Thought process disorder in schizophrenia: The listerner's task». Brain \& Language, 1977, 4, 95-114.

Rosenberg, S., y TUCKer, G. J.: «Verbal behavior and schizophrenia. The semantic dimension». Arch. of Gen. Psychiat., 1979, 36, 1331-1337.

Rosvold, H. E.; Mirsky, A.; Sarason, I.; Bransome, E. D., y Beck, L. H.: «A continuous performance test of brain damage». J. of Consulting Psychology, 1956, 20, 343-350.

RuIZ-VARGAS, J. M.: «Procesamiento de la información en la esquizofrenia: Hacia una interpretación alternativa». Estudios de Psicología, 1985, 22, 33-51.

RuIz-VARgaS, J. M.: Esquizofrenia: Un enfoque cognitivo. Madrid: Alianza Psicología, 1987.

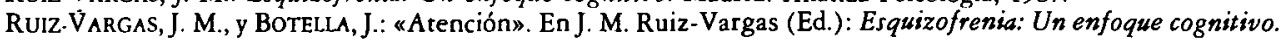
Madrid: Alianza Psicología, 1987.

Salzinger, K.; Portnoy, S., y Feldman, R. S.: "Verbal behavior of schizophrenic and normal subjects». Annals of the New York Academic of Sciences, 1964, 105 (15), 845-860.

Salzinger, K.; Portnoy, S., y Feldman, R. S.: "Communicability deficit in schizophrenics resulting from a more general deficit». En S. Schwartz (Ed.): Language and cognition in schizophrenia. Nueva York: LEA, $1978,35-53$.

Salzinger, K.; Portnov, S.; Feldman, R. S., y Patenaude-Lane, J.: «From method to madness: The cloze procedure in the study of psychopathology». En W. Rieber (Ed.): Applied Psycholinguistics and mental bealth. Nueva York: Plenum Press, 1980, págs. 94-113. 
ScINTo, L. F. M.: «Textual competence: A preliminary analysis of orally generated texts». Linguistics, 1977, 194, 5-34.

SCINTO, L. F. M.: The acquisition of functional composition strategies for text. Hamburgo: Helmut Buske Verlag, 1982.

Scinto, L. F. M.: «The architectonics of texts produced by children and the development of higher cognitive functions». Discourse Processes, 1984, 7, 371-418.

SCHNEIDER, W., y SCHIFFRIN, R. M.: «Controlled and automatic human information processing: I. Detection, search and attention». Psychological Review, 1977, 84, 1-66.

SEGLAS, J.: Des troubles du langage chez les aliénés. París: Rueff et Cie., 1982.

SCHIFFRIN, R. M., y SCHNEIDER, W.: "Controlled and automatic human information processing: Il. Perceptual learning, automatic attending, and a general theory». Psychological Review, 1977, 84, 127-190.

Siegel, A.; HARRow, M.; Reilly, F. E., y TUCKer, G. T.: «Loose associations and disordered speech patterns in chronic schizophrenics». J. of Nerv. and Mental Dis., 1976, 162, $105-112$.

STEEDMAN, M. J., y JOHNSON-LaIRD, P. N.: «The production of sentences, utterances and speech acts: Have computers anything to say?». En B. Butterworth (Ed.): Language Production. Vol. 1. Londres: Academic Press, 1980, págs. 111-141.

Suluvan, H. S.: «Peculiarity of thought in schizophrenia». Amer. J. of Psychiat., 1925, S, 21-86.

TUCKER, G. J., y ROSENBERG, S. D.: «Computer content analysis schizophrenic speech: A preliminary report». Amer. J. of Psychiat., 1975, 132, 611-616.

VAN DIJK, T. A.: «Recalling and summarizing complex discourse». En W. Burghardt y K. Hölker (Eds.): Text processing. Berlín: Walter de Gruyter, 1979.

VAN DIJK, T. A.: Macrostructures: An interdisciplinary study of global structures in discourse, interaction and cognition. Hillsdale, N. J.: LEA, 1980.

VAN Dijk, T. A.: La ciencia del texto. Paidós Comunicación, 1984, (Ed. or., 1978). VAN Dijk, T. A. (Ed): Handbook of discourse analysis. Londres: Academic Press, 1985, (4 vols.).

ZAMMUNER, V. L.: Speech production. Strategies in discourse planning: A theoretical and empirical inquiry. Hamburgo: Helmur Buske Verlag, 1981.

Zavarin, V.: «Frame analysis of schizophrenic discourse». Proceedings of the Third Annual Meeting of the Berkeley Linguistic Society. Vol. 3. Berkeley: BLS, 1981, 545-558.

ZAVARIN, V.: «Modification of goal directed behavior in discourse». En R. F. Thompson, L. H. Hicks y V. B. Shuyrkov (Eds.): Neural mechanisms of goal directed bebavior and learing. Nueva York: Academic Press. 1980.

ZUBIN, J., y SPRING, B.: «Vulnerability: A new view of schizophrenia». J. of Abnormal Psychology, 1977, 86, págs. 103-126. 